\title{
In Silico Modeling of Magnetic Resonance Flow Imaging in Complex Vascular Networks
}

\author{
Krzysztof Jurczuk*, Marek Kretowski, Pierre-Antoine Eliat, Herve Saint-Jalmes, and Johanne Bezy-Wendling
}

\begin{abstract}
The paper presents a computational model of magnetic resonance (MR) flow imaging. The model consists of three components. The first component is used to generate complex vascular structures, while the second one provides blood flow characteristics in the generated vascular structures by the lattice Boltzmann method. The third component makes use of the generated vascular structures and flow characteristics to simulate MR flow imaging. To meet computational demands, parallel algorithms are applied in all the components. The proposed approach is verified in three stages. In the first stage, experimental validation is performed by an in vitro phantom. Then, the simulation possibilities of the model are shown. Flow and MR flow imaging in complex vascular structures are presented and evaluated. Finally, the computational performance is tested. Results show that the model is able to reproduce flow behavior in large vascular networks in a relatively short time. Moreover, simulated MR flow images are in accordance with the theoretical considerations and experimental images. The proposed approach is the first such an integrative solution in literature. Moreover, compared to previous works on flow and MR flow imaging, this approach distinguishes itself by its computational efficiency. Such a connection of anatomy, physiology and image formation in a single computer tool could provide an in silico solution to improving our understanding of the processes involved, either considered together or separately.
\end{abstract}

Index Terms-Computational fluid dynamics (CFD), computational modeling, magnetic resonance imaging (MRI), parallel computing, vascular network.

\section{INTRODUCTION}

C OMPUTATIONAL models play a very important role in science and industry. In the medical field, they are used to reproduce biological systems and equipment by using and pro-

Manuscript received May 10, 2014; accepted June 14, 2014. Date of publication July 09, 2014; date of current version October 28, 2014. This work was supported in part by Polonium 2012-2013 (French-Polish cooperation program) under the project "MRI analysis and modeling for tissue characterization" and in part by Bialystok University of Technologyunder Grant S/WI/2/2013 and Grant W/WI/2/2014. Asterisk indicates corresponding author.

*K. Jurczuk is with the Faculty of Computer Science, Bialystok University of Technology, 15-351 Bialystok, Poland (e-mail: k.jurczuk@pb.edu.pl).

M. Kretowski is with the Faculty of Computer Science, Bialystok University of Technology, 15-351 Bialystok, Poland.

P. A. Eliat is with Universite de Rennes 1, PRISM, F-35000 Rennes, France, and with CNRS, UMS 3480, F-35000 Rennes, France, and also with INSERM, UMS 018, F-35000 Rennes, France.

H. Saint-Jalmes is with INSERM, U1099, F-35000 Rennes, France, and with Universite de Rennes 1, LTSI, F-35000 Rennes, France, and is also with, Centre Eugene Marquis, F-35000 Rennes, France.

J. Bezy-Wendling is with INSERM, U1099, F-35000 Rennes, France, and with Universite de Rennes 1, LTSI, F-35000 Rennes, France.

Color versions of one or more of the figures in this paper are available online at http://ieeexplore.ieee.org.

Digital Object Identifier 10.1109/TMI.2014.2336756 gramming mathematical formulas that describe physical processes usually in a simplified manner [1]. This artificial representation of reality allows us to perform various experiments in silico without disturbing the actual system. The advantage of using models in also evident when significant number of scenarios are to be tested. Simultaneous simulations are often the only way to evaluate various combinations of parameters.

In this paper, we deal with the modeling of magnetic resonance imaging (MRI) of vascular structures. Such imaging, called in medicine magnetic resonance angiography (MRA) [2], is influenced not only by the standard contrast parameters of tissues (i.e., relaxation times and proton density) but also by blood behavior, e.g., flow direction, velocity and patterns. In one respect, pathology detection and characterization can be improved by using the intrinsic flow sensitivity of MRI. For example, flow-related signal diminution can be identified in zones of abnormal vessel widening (e.g., aneurysm [4]) or narrowing (e.g., stenosis [5]). Such vessel perturbations can lead to many serious consequences, such as cerebral hemorrhage [6], liver ischemia with hepatic insufficiency [7], or thromboembolic pulmonary embolism [8]. Another example is the use of contrast agents transported by blood to enhance the MR signal in hypervascularized areas of tumoral lesions (contrast-enhanced MRA) [3].

In other respects, flow during MRI acquisition can give rise to various image artifacts (as other movements) [9]-[12]. These flow-related artifacts appear in the regions with complex vascular structures as well as simple straight vessels. They lead to additional difficulties in image analysis which can lead to image misinterpretation and inappropriate patient treatment. Hence, the understanding of MR flow image formation is of importance, due to clinical assessment of the disease as well as problems with artifacts. The literature includes many studies, both experimental and numerical, to predict the influence of flow on MRI, e.g., [11], [13]-[18] to name a few.

MR flow imaging is a complicated process that involves many factors linked to anatomy, physiology, and imaging modalities. MRI sequence events (e.g., excitation, spatial encoding, etc.) are located in time over ranges usually from a few milliseconds to a few seconds. Due to the flow, magnetized fluid particles are transported during and between those MRI events and are thus subjected to constantly changing magnetic conditions [9]. Therefore, fluids in images can be visible in a totally unpredictable way. Evidently, this visibility depends on the flow pattern (e.g., turbulent, steady) and the flow characteristics, such as velocity and direction. In turn, the spatial and temporal flow behavior results from the topology, geometry, and physiology of the vascular structures. Even small changes 


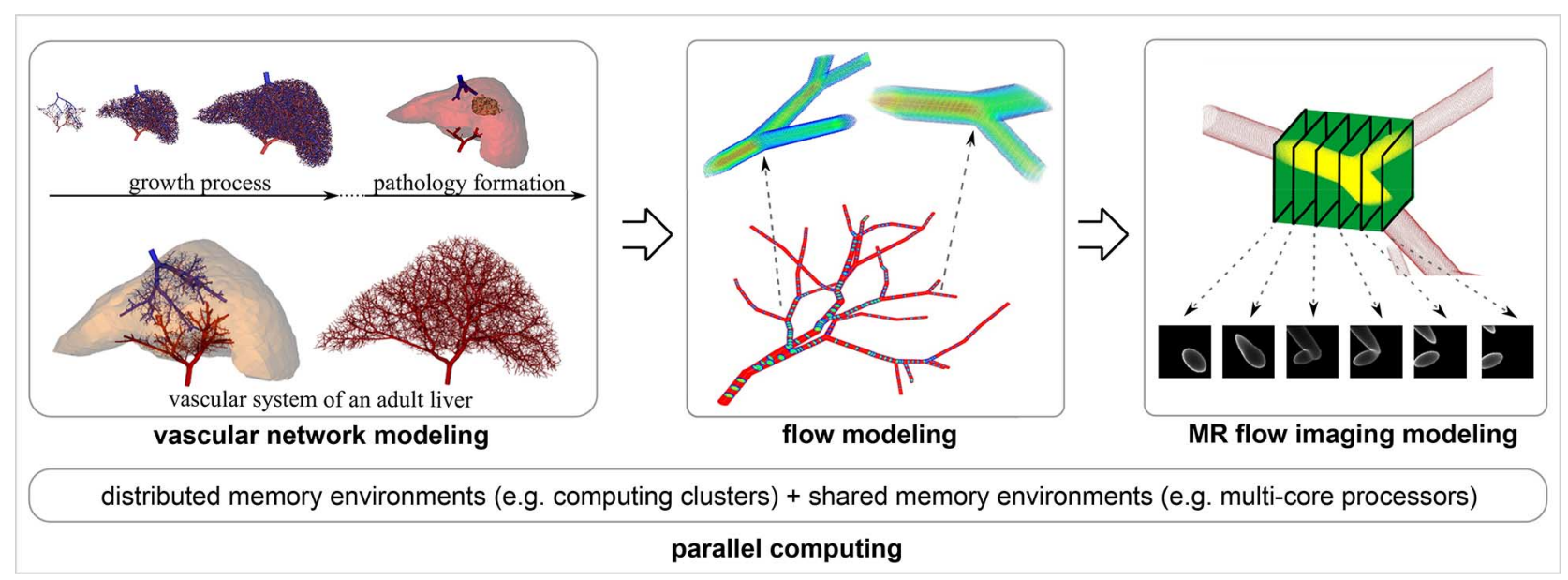

Fig. 1. General overview of the three-component computational model. The first component provides vascular geometries based on physiological and hemodynamics parameters. The second one carries out flow simulations, while the third one reproduces MR flow imaging processes. To deal with complex vascular structures (e.g., hundreds and thousands of vessels), parallel algorithms are developed in each model component.

in vessel geometry or physiological parameters (e.g., pressure, blood flow) can substantially change the fluid behavior and may impact the risk of later severe clinical consequences [19], [20].

These interdependencies between anatomy, physiology, and image formation in clinical treatment of diseases was the motivation to create an MRI model that integrates three components in one tool, i.e., a vascular geometry generator, a flow simulator and a virtual scanner of MR flow imaging (see Fig. 1). The general aim of this integrative model is to provide an in silico solution to improve our understanding of all these processes taken together. During the acquisition of clinical images, it is usually impossible to control and monitor all the involved processes/events in each step of the image formation. By contrast, such a computational model may represent a noninvasive way to obtain some detailed insight and control parameters that would be difficult or even impossible to access in real experiments.

The original part of the works presented in this paper is an efficient and effective integration of three model components that have been shown to be successful when applied separately: generation of a synthetic vascular network [21], the lattice Boltzmann method (LBM) [28], and numerical simulations of MR flow imaging [35]. First of all, the effective sharing of data between model components requires additional developments, such as automatic vessel discretization. The second issue concerns the development of computationally efficient algorithms suitable for the investigation of complex vascular structures. Besides addressing questions about the integrative model quality and the level of its detail, the challenge of such an approach lies also in the demand for high performance computing to perform simulations within a reasonable period of time. This has imposed the use of parallel computing to implement the proposed algorithms, and this aspect also represents another original contribution of this work. In all the components of the model, computations are adapted to be performed at various parallel architectures (e.g., using shared-memory, distributed-memory, and hybrid machines). The main goal of this paper is to present the model and its possibilities at different levels (and scales). To our knowledge, the proposed tool is currently the only solution available in the literature that simultaneously treats the modeling of vascular geometries, flow behavior, and MR flow imaging.

The first component of the model (see left part of Fig. 1) is used for vascular network generation. The structure of the vascular network is obtained through the process of vascular development associated with tissue growth. The tissue growth is modeled by progressive creation of new macro-cells (parts of tissue with capillaries inside) [21]. The vascular network can consist of several vascular trees (e.g., arterial and venous trees) that are connected in terminal vessels by macro-cells. Many physiological and functional parameters of the tissue, as well as geometrical and hemodynamic parameters, can be modified to adapt to a specific vascularization, e.g., for liver or kidney. Because we consider large three-dimensional (3D) vascular trees made up of thousands and more vessels and bifurcations, parallel algorithms were also developed for vascular development [22]. As far as we know, in all previous approaches of vascular modeling (e.g., [23]-[27]), only sequential algorithms were applied to generate vascular trees.

The second component of the model (see middle part of Fig. 1) applies LBM to simulate flow behavior in vascular structures generated by the first component. LBM is a kinetic-based approach known for its computational efficiency and easy accommodation to complex geometries. At the same time, it is able to produce results that obey the Navier-Stokes equations to a high level of accuracy [29], [30]. This method of computational fluid dynamics (CFD) allowed us to carry out simulations in hundreds and thousands of 3-D vessels. On the other hand, in the most advanced previous studies on the modeling of flow in 3-D vascular structures [31], [32], tens and hundreds of vessels were examined with the use of huge computational resources (tens of thousands of processors distributed between several research centers).

The third component of the model (see right part of Fig. 1) reproduces MR imaging processes using geometries and flow maps that are both provided by the two previous components. The imaged area is divided into cubic elements in such a way that each element contains information about the flow characteristics of the fluid filling them. The influence of MRI events 
is tracked in each cubic element by the discrete time solution of the Bloch equation [33] in the form of rotation matrices and exponential scaling [34]. At the same time moments, the flow influence is taken into account by the algorithm of magnetization transport between cubic elements [35]. Finally, the image is directly constructed by sampling the resulting MR signal in the k-space matrix and Fourier transform processing. Such an approach allows us to closely follow the physical process of MRI, along with the automatic incorporation of flow-related artifacts. Thus, there is no need for geometrical procedures for mapping the physical plane to the image plane, such as mesh transformation [16]. Flow is automatically considered during most MRI events, e.g., during excitation, signal acquisition, and spatial encoding. Moreover, the model component stands out by its low computational load, and parallel implementation that makes it possible to consider complex vascular networks. Previous works (e.g., [13], [14], [16], [18]) considered one vessel at a time or a single bifurcation at most.

The rest of the paper is organized as follows. Section II describes the model components and how they are connected. In Section III, the proposed approach is verified. First, we confront the simulation results with in vitro experiments. Then, the simulation possibilities of the model in complex vascular structures are shown and inspected. Section IV presents the evaluation of computational performance of the model. Section IV provides a discussion along with a summary and possible future research directions.

\section{MODEL DESCRIPTION}

\section{A. Vascular Network Modeling}

The vascular network component is composed of two elements: tissue and vascular trees [21]. The tissue is represented by a set of macroscopic functional units (MFU, also called macro-cells, with a typical size $0.1-1 \mathrm{~mm}^{3}$ ) that fill a predefined organ shape. The vascular trees supply MFUs with blood. Moreover, in each MFU a micromodel is hidden [3]. It represents the microvascularization responsible for contrast agent propagation in the tissue. The micromodel is built of few compartments used to represent the arterioles, venules, sinusoidal network (discontinuous capillaries and pores), and interstitial fluid. Blood flows from the arterioles to the capillaries, then enters the interstitial liquid compartment and leaves the MFU via the venules. In this paper, we focus on the vascular network model at the macroscopic level, since it is subsequently coupled with the flow and MRI models. For a detailed survey of the complete model, we refer the reader to our previous papers, e.g., [21], [3].

The vascular tree is composed of vessels that can divide to create bifurcations (Fig. 2) [21]. Each vessel segment (part of the vessel between two successive bifurcations) is represented by an ideal rigid tube with a fixed radius, wall thickness, length, and position. The geometry of the smallest vessels, i.e., capillaries, is not considered as they are hidden in the MFUs. Based on a morphometrical investigation dealing with larger vessels, e.g., conducted by Zamir [36], it is assumed that each vascular tree forms a binary tree (Fig. 2). As a result, we do not take

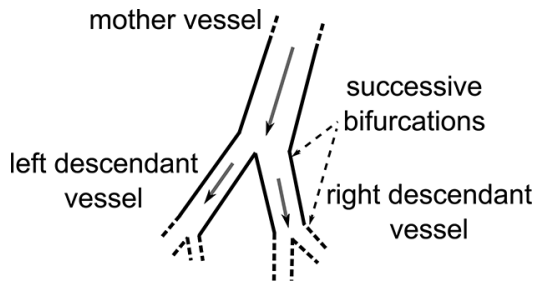

Fig. 2. Part of a binary vascular tree. Mother vessel and its two descendant vessels (left and right) connected by a bifurcation.

account of anastomoses (e.g., mutual vessel intersections) that occur mainly among vessels with very small radii.

The parameters of the vessels (i.e., blood flow, pressure, radius, etc.) are calculated according to the basic laws of physics. In brief, the first constraint deals with the decreasing radii of the vessels in successive bifurcations, creating a relationship between the radius of a mother vessel and the radii of its two descendants [37]. Secondly, the mass conservation law must be observed at each bifurcation, i.e., the quantities of blood entering and leaving a bifurcation have to be equal. The other unknowns are constrained by treating blood as a Newtonian (constant viscosity) incompressible fluid exhibiting laminar flow governed by Poiseuille's law [38].

The structure of the vascular trees is generated using the algorithm of vascular development driven by progressively increasing the number of MFUs. The model is initialized by means of a vascular network consisting of only a few vessel segments and MFUs (e.g., based on segmented images or anatomical data). The organ shape can be bounded by reconstruction from CT scans, MRI images or even manually. The simulation starts with an organ whose size is a fraction of the adult one. In discrete time moments, the organ is enlarged until it reaches its full mature form. In consecutive algorithm cycles, each MFU can either divide and give rise to a new MFU or die (analogy with mitosis and necrosis processes [39]). Since mitosis is predominant during the growth phase, the increasing blood requirements of the growing tissue induce development of the vascular network. New MFUs fill empty spaces that appear during the organ growth phase.

The newly appearing MFUs are initially not perfused (connected) by the existing vascular network. In reality, such ischemic parts of the tissue produce angiogenic factors which stimulate the neighboring vessels to sprout new branches able to supply blood. In the model, a fixed number of the neighboring vessels (candidates to perfusion) is found for each new MFU. Then, each candidate vessel creates a new bifurcation that is used to temporarily perfuse the MFU. Finally, the optimal configuration of candidate vessel segments (with the lowest sum of volumes) is chosen as the permanent configuration. Subsequently, the vessels' characteristics (i.e., blood flow, pressure, etc.) are recalculated to satisfy all the physical constraints used.

For each MFU a class is assigned. The MFU class determines most of its structural and functional properties (e.g., size, probability of mitosis/necrosis) as well as its physiological features (e.g., blood pressure, blood flow rate). The MFU class can also change over time, which makes it possible to simulate pathology formation and evolution. 
In our previous studies, the vascular model was successfully adapted to virtual representations of two internal organs: liver [40] and kidney [41]. It should be emphasized that these representations were oriented towards image generation and, therefore, our attention was focused on vessel networks that play a very important role in clinical imaging.

1) Parallel Algorithm for Vascular Network Modeling: The perfusion of MFUs in the vascular network is the most time-consuming part of the vascular development algorithm. This is because it is necessary to create and test several temporary bifurcations for each new MFU before finding the optimal configuration. This requires many calculations to satisfy the imposed constraints related to the geometry and hemodynamics at the level of the bifurcation as well as the vascular network. Thus, both of the developed parallel algorithms [42], [22] are focused on this process.

The first parallel algorithm [42] is based on the master-slave model [43] and uses the message passing paradigm [44]. The newly appearing MFUs are distributed by a master processor among slave processors (functional/task decomposition [43]). Each slave processor attempts to find optimal bifurcations for its assigned MFUs. Each time the search ends successfully, a slave sends the parameters of the optimal bifurcation to the master processor. The master processor is responsible for managing the permanent perfusion process. It searches among its vascular structures for vessels that are proposed by the slave processor to perfuse the considered MFU. If it cannot find at least one of these vessels, then the MFU is rejected. Such a situation simply means that this particular vessel (or vessels) has been used earlier to perfuse another tissue element and was replaced by new vascular structures. The rejected MFU leaves an empty space in the tissue for other new MFUs that can appear during the next subcycle. However, in the case of acceptance, the MFU is permanently connected to the vascular system. Then, information about the permanent changes in vascular trees is broadcasted by the master processor to the slaves. Most of the communication between processors is carried out in a nonblocking mode to facilitate the overlapping of computation with communication. Moreover, several load balancing algorithms are proposed to obtain an equal spreading of load over the processors.

The second parallel algorithm [22] applies the shared address space paradigm [45]. Each time a new MFU appears or an existing one dies, all the calculations in different vascular trees (e.g., hepatic arteries, portal veins, or hepatic veins in the case of liver) are divided between different processors (functional/task decomposition). For instance, each new MFU is connected to the vascular network simultaneously in all the vascular trees. The advantage of this approach is that no explicit communication between processors is needed. However, the speedup of this approach is limited by the number of vascular trees. It is also possible to couple the two parallelizations to obtain a hybrid algorithm [46] that is able to exploit efficiently computing clusters equipped with shared- and distributed-memory architectures.

\section{B. Flow Modeling}

In order to study hemodynamics in the modeled complex vascular networks, the used CFD approach should have relatively

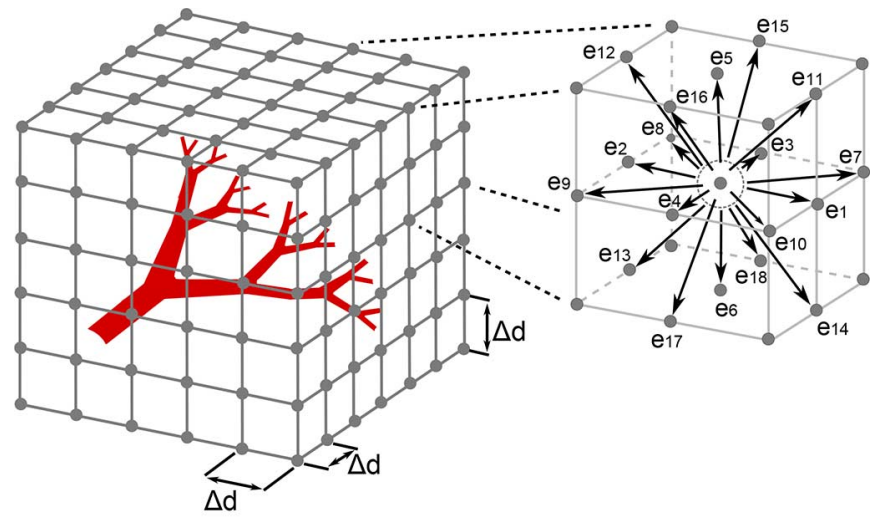

(a)

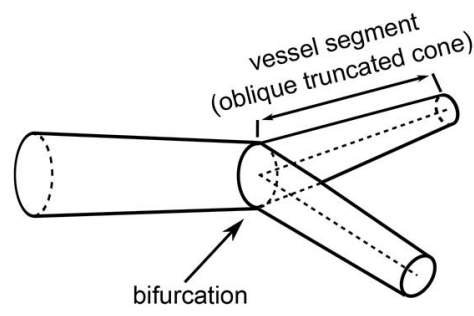

(b)

Fig. 3. Space discretization: (a) lattice of nodes connected by fixed paths, 3-D model with 19 discrete velocities $\left(\mathbf{e}_{i}\right)$ (19 paths to neighbors), sphere in the center denotes the velocity equals zero $\left(\mathbf{e}_{0}\right)$ (i.e., for nonmoving particles); (b) vessel segments represented by oblique truncated cones.

low computational requirements. An ideal candidate is the lattice Boltzmann method (LBM) [28]. This method has been extensively developed over the last decade, and, as a result, has become a powerful alternative to the conventional CFD solutions based on Navier-Stokes equations [47]. The advantages of LBM include not only simple arithmetic calculations and highly efficient parallelization, but also easy formulation of boundary conditions and high applicability to complex geometries, which stem from the bridging between micro- and macroscale physics. It has been demonstrated that the Navier-Stokes equations can be derived from the LBM equations for incompressible flow at low Mach number [48], [49]. In other cases, LBM has been shown to be a robust approximation of the Navier-Stokes equations [50], [51].

LBM solves the Boltzmann transport equation [52] in discretized space and time. The space is represented by a regular finite lattice of nodes connected by fixed paths [Fig. 3(a)]. The fluid dynamics at the nodes is described by motion of fictitious mesoscopic particles that can move to neighboring nodes (streaming step), and then collide and relax (collision step). These two steps are performed by tracking the particle distribution function $f_{i}(\mathbf{r}, t)$ in time $t$ at each node. $i$ is the streaming direction along one of the fixed paths between neighboring nodes and $\mathbf{r}$ is the node position defined as: $\mathbf{r}=x \hat{\mathbf{i}}+y \hat{\mathbf{j}}+z \hat{\mathbf{k}}$, where $\hat{\mathbf{i}}, \hat{\mathbf{j}}, \hat{\mathbf{k}}$ are unit vectors in $x, y, z$ directions. At the collision step, the distributions $f_{i}(\mathbf{r}, t)$ relax to the equilibrium state $f_{i}^{e q}(\mathbf{r}, t)$. At the streaming step, the distributions $f_{i}(\mathbf{r}, t)$ stream with their discrete velocities $\mathbf{e}_{i}$ along the $i$ direction to the neighboring nodes at $\mathbf{r}+\Delta \mathbf{r}_{i}$ positions $\left(\Delta \mathbf{r}_{i}=\mathbf{e}_{i} \Delta d / c\right.$ is the streaming distance, $\Delta d$ is the distance between nearest-neighboring nodes 
and $c$ is the lattice constant related to the propagation factor on the lattice and is usually set to unity [28]). This two-step evolution is expressed by the lattice Boltzmann equation in the following way:

$$
\begin{aligned}
& f_{i}\left(\mathbf{r}+\Delta \mathbf{r}_{i}, t+\Delta t\right)-f_{i}(\mathbf{r}, t)=\Omega_{i}(\mathbf{r}, t), \\
& \Omega_{i}(\mathbf{r}, t)=1 / \tau\left(f_{i}^{e q}(\mathbf{r}, t)-f_{i}(\mathbf{r}, t)\right)
\end{aligned}
$$

where $\Delta t$ is the time step and $\tau$ is the dimensionless relaxation time related to fluid viscosity. The collision term $\Omega$ is approximated by the broadly accepted and computationally efficient single-relaxation-time Bhatnagar-Gross-Krook (BGK) operator [53].

Different lattice topologies have been developed for LBM. They are classified using the DxQy notation, where $x$ is the spatial dimension and $y$ is the number of discrete velocities. There are three preferred topologies for 3-D cubic lattices: D3Q15, D3Q19, and D3Q27. With increasing number of discrete velocities the accuracy of the solution also increases. However, there is also an increase in computation time, number of iterations, code complexity and memory consumption. It has been shown that the difference between D3Q19 and D3Q27 in fluid flow simulations is not necessarily significant [54]. On the other hand, D3Q15 topology can exhibit velocity oscillations and is prone to computational instability, especially at higher Reynolds numbers. Thus, in the proposed approach, we use the D3Q19 topology [55] [Fig. 3(a)] for which the equilibrium distribution function takes the following form:

$$
f_{i}^{e q}=\rho \omega_{i}\left[1+\frac{3}{c^{2}} \mathbf{e}_{i} \cdot \mathbf{u}+\frac{9}{2 c^{4}}\left(\mathbf{e}_{i} \cdot \mathbf{u}\right)^{2}+\frac{3}{2 c^{2}} \mathbf{u} \cdot \mathbf{u}\right]
$$

where $\rho \mathbf{u}=\sum_{i=0}^{18} \mathbf{e}_{i} f_{i}$ is the macroscopic lattice velocity, $\rho=\sum_{i=0}^{18} f_{i}$ is the dimensionless lattice density, $\omega_{i}$ is the weighting factor $\left(\omega_{0}=1 / 3, \omega_{1 . .6}=1 / 18, \omega_{7.18}=1 / 36\right)$. The lattice velocity $\mathbf{u}$ is interpreted as the fraction of the distance between neighboring nodes through which the fluid travels per time step $\Delta t$. Thus, the physical macroscopic velocity is obtained by multiplying the lattice velocity by $\Delta d / \Delta t$.

The following boundary conditions are applied. At the entrances and exits (i.e., inflow and outflow points) of the geometry, it is possible to impose the constant velocity or pressure boundary conditions. The unknown values of the distribution function are calculated by the extrapolation method proposed by Guo et al. [56]. As regards walls, we use the procedure called the bounce-back scheme for the no-slip (i.e., zero velocity) boundary condition [55].

To couple the vascular network component with the lattice Boltzmann algorithm, the analytical definition of vessel segment shape (i.e., radius, beginning and end points) is changed to a discrete representation. We chose to represent each vessel segment by a lattice of nodes inside a 3-D volume similar to an oblique truncated cone, to provide a continuous shape (without jumps) at bifurcations [Fig. 3(b)]. The algorithm to check whether a lattice node is inside or outside such a volume was designed (see Fig. 4 for its visualization). The algorithm inputs are the beginning/end points and radii of the considered (as well as the preceding) vessel segments. The algorithm steps are as follows.

1) Point $N$ is projected along the line $X Y$ (axis of the considered vessel segment) to meet two lines; the first line is perpendicular to the axis of the preceding vessel segment and passes through point $X$; the second line is perpendicular to the axis of the considered vessel segment and passes through point $Y$.

2) Based on the points $A$ and $B$ so obtained as well as the considered vessel segment data (radii $|A 1 X|,|B 1 Y|$ and beginning/end points $X / Y$ ), we find points $A 1, A 2$ and $B 1, B 2$.

3) Points $P 1, P 2, P 3, P 4$ are found by projecting the point $N$ to lines $A 1 A 2$ and $B 1 B 2$ along lines $A 1 B 1$ and $A 2 B 2$.

4) Finally, the point $N$ is inside the considered vessel segment if at least two points out of $P 1, P 2, P 3, P 4$ are inside the segment $A 1 A 2$ or $B 1 B 2$; the distance from point $X$ is checked for points $P 1$ and $P 2$, while the distance from point $Y$ is verified for points $P 3$ and $P 4$.

In the 3-D version of the algorithm, the initial projections have to be performed on two planes. The first plane is perpendicular to the axis of the preceding vessel segment and passes through point $X$. The second plane is perpendicular to the axis of the considered vessel segment and passes through point $Y$. The remaining steps are the same.

1) Parallel Algorithm for Flow Modeling: The LBM requires many floating point values to store and operate on the particle distribution function in each lattice node. For the applied D3Q19 lattice topology, at least $19 * 2$ floating point values are needed for each lattice node. Since we consider complex vascular structures with many vessel segments of different sizes, sparse filling of fluid compared to (surrounding) solid tissue will be usually observed. Thus, it is wasteful to use solid nodes (located outside the fluid) during flow simulations. For this reason, an additional list (vector) of the fluid points is created in the model. This list is used by the flow algorithm (so-called sparse lattice representation with neighborhood indirect addressing [57]). For solid nodes only their positions are stored. The parallel flow algorithm is based on previous LBM parallelization studies [57], [58] that exploit this type of data representation.

The list of fluid nodes is divided equally between processors (data decomposition [43]) leading to balanced workload among processors [58]. Each processor is able to perform the collision step independently at all assigned nodes, because this operation needs only spatially local data. Three situations may occur regarding the streaming step. If the neighboring node is located on a wall or is assigned to the same processor, the new value of the particle distribution function is streamed in the same way as in the sequential algorithm. Otherwise, when the neighboring node is located at a different processor, the necessary inter-processor communication is realized by the message passing paradigm. Additional vectors of ghost (halo) nodes are created to ensure efficient handling of sending and receiving operations. These nodes store data to be sent to other processors as well as data received from other processors. Thus, they do not participate in the collision step. 


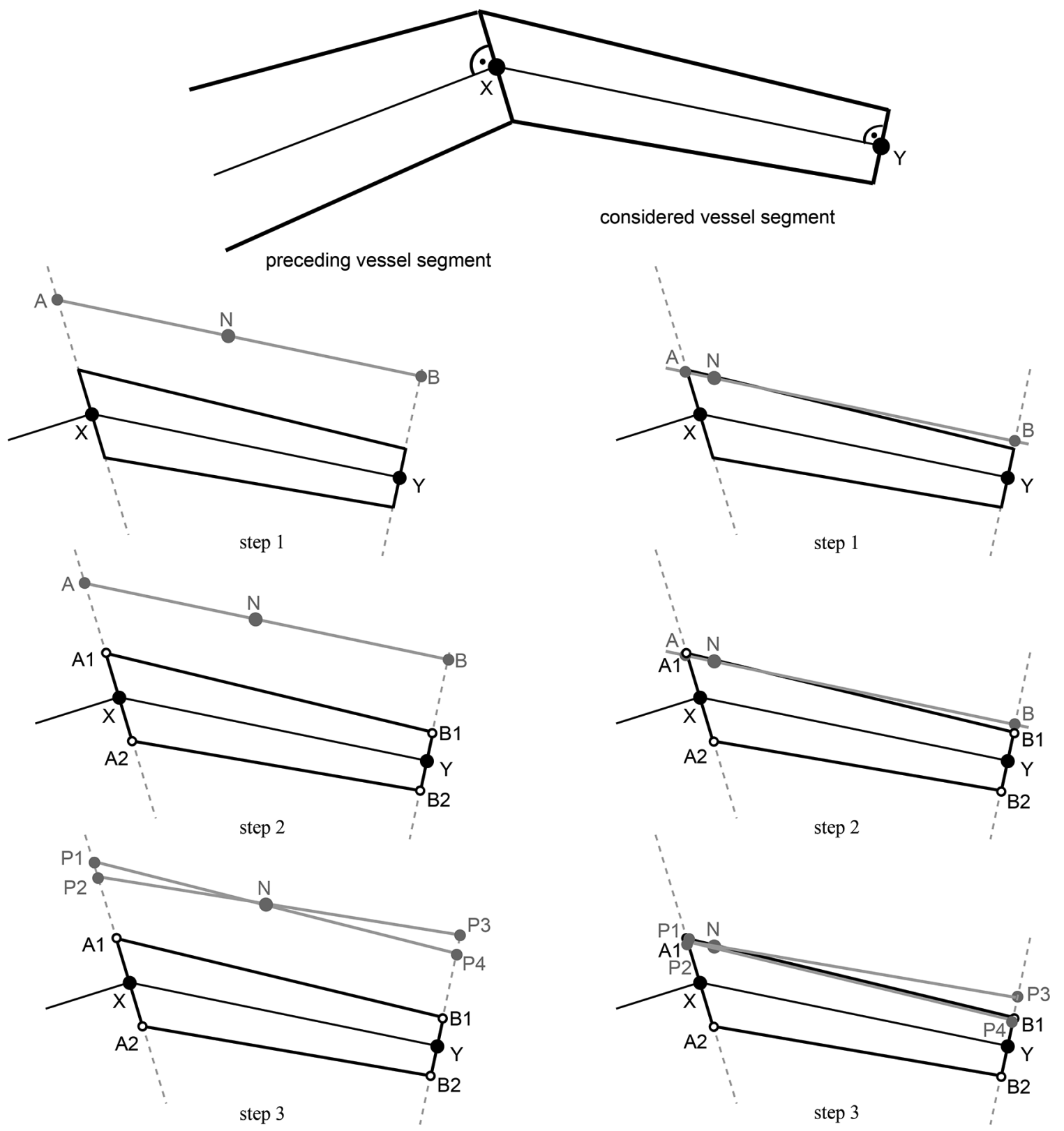

Fig. 4. Visualization of the algorithm to check whether the point/node $N$ is inside the vessel segment $A 1 A 2 B 1 B 2$. In images on left, the point $N$ is outside the considered vessel segment. In images on right, the point $N$ is situated in such a way that it is inside the considered vessel segment, but it would be outside for the vessel segment with constant radius (for cylindrical vessel in 3-D).

\section{Modeling of MR Flow Imaging}

The imaged area is divided into cubic elements in such a way that one LBM grid node is situated at the center of each cubic element. This kind of discretization guarantees that each cubic element contains information about the flow of the fluid filling it (from the flow simulation) as well as the MR characteristics (proton density and relaxation times). For stationary tissue structures, the flow velocity equals zero.

The whole MR imaging process is divided into sufficiently small time steps $\Delta t$ [35]. After each time step, the magnetization vector $\mathbf{M}=M_{x} \hat{\mathbf{i}}+M_{y} \hat{\mathbf{j}}+M_{z} \hat{\mathbf{k}}$ in each cubic element is modified. The new magnetization $\mathbf{M}(\mathbf{r}, t+\Delta t)$ is calculated taking into account both the flow influence $\Delta \mathbf{M}_{F}$ and the imaging process $\mathbf{A}_{\mathrm{MRI}}$ by means of the following equation (see Fig. 5):

$$
\mathbf{M}(\mathbf{r}, t+\Delta t)=\mathbf{A}_{\mathrm{MRI}}(\mathbf{r}, \Delta t)\left[\mathbf{M}(\mathbf{r}, t)+\Delta \mathbf{M}_{F}(\mathbf{r}, \Delta t)\right]
$$

where $\mathbf{r}$ is the spatial position of the considered cubic element.

The flow influence is realized by the propagation (transport) of magnetization between cubic elements. The magnetization changes in a cubic element at position $\mathbf{r}$ in time step $\Delta t$ are expressed as follows:

$$
\Delta \mathbf{M}_{F}(\mathbf{r}, \Delta t)=\Delta \mathbf{M}_{\mathrm{IN}}(\mathbf{r}, \Delta t)-\Delta \mathbf{M}_{\mathrm{OUT}}(\mathbf{r}, \Delta t) .
$$




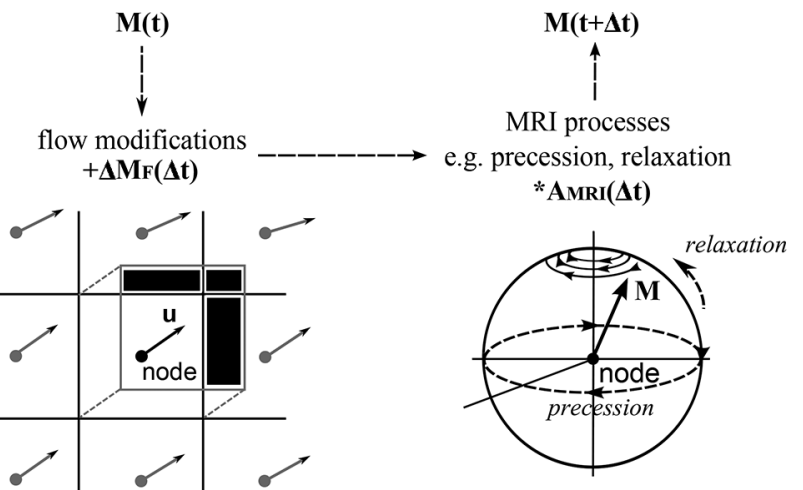

magnetization transport

between neighboring nodes/elements magnetization evolution in each node/element independently

Fig. 5. Coupling the flow and MRI algorithms. At first, magnetization in each cubic element is modified based on the flow characteristics, taking into account the magnetization transfer between neighboring nodes. Then, MRI processes are modeled. In each cubic element, a magnetization vector is changed according to the Bloch equation.

The magnetization values entering $\Delta \mathbf{M}_{\mathrm{IN}}$ and leaving $\Delta \mathrm{M}_{\mathrm{OUT}}$ for each cubic element are calculated based on the velocity $\mathbf{u}=u_{x} \hat{\mathbf{i}}+u_{y} \hat{\mathbf{j}}+u_{z} \hat{\mathbf{k}}$ obtained from the flow model component. For sake of clarity, the equations of $\Delta \mathbf{M}_{\mathrm{IN}}$ and $\Delta \mathbf{M}_{\mathrm{OUT}}$ are shown in the full form for the two-dimensional (2-D) case. The fractions of magnetization entering a cubic element are calculated based on its flow properties and magnetizations of its neighboring elements

$$
\begin{aligned}
\Delta \mathbf{M}_{\mathrm{IN}}(\mathbf{r}, \Delta t) \\
=\mathbf{M}\left(\mathbf{r}-\Delta d \frac{u_{x}(\mathbf{r})}{\left|u_{x}(\mathbf{r})\right|} \hat{\mathbf{i}}-\Delta d \frac{u_{y}(\mathbf{r})}{\left|u_{y}(\mathbf{r})\right|} \hat{\mathbf{j}}, t\right)\left|u_{x}(\mathbf{r})\right|\left|u_{y}(\mathbf{r})\right| \\
\quad+\mathbf{M}\left(\mathbf{r}-\Delta d \frac{u_{x}(\mathbf{r})}{\left|u_{x}(\mathbf{r})\right|} \hat{\mathbf{i}}, t\right)\left|u_{x}(\mathbf{r})\right|\left(1-\left|u_{y}(\mathbf{r})\right|\right) \\
\quad+\mathbf{M}\left(\mathbf{r}-\Delta d \frac{u_{y}(\mathbf{r})}{\left|u_{y}(\mathbf{r})\right|} \hat{\mathbf{j}}, t\right)\left(1-\left|u_{x}(\mathbf{r})\right|\right)\left|u_{y}(\mathbf{r})\right|
\end{aligned}
$$

The fractions of magnetization leaving a cubic element are calculated from its flow properties and its magnetization value (see "black rectangles" in Fig. 5)

$$
\begin{aligned}
& \Delta \mathbf{M}_{\text {OUT }}(\mathbf{r}, \Delta t)=\mathbf{M}(\mathbf{r}, t)\left[\left|u_{x}(\mathbf{r})\right|\left|u_{y}(\mathbf{r})\right|\right. \\
& \left.\quad+\left|u_{x}(\mathbf{r})\right|\left(1-\left|u_{y}(\mathbf{r})\right|\right)+\left(1-\left|u_{x}(\mathbf{r})\right|\right)\left|u_{y}(\mathbf{r})\right|\right] .
\end{aligned}
$$

In 3-D modeling, each sum component in these equations is made up of two cases, accordingly, with $\left|u_{z}(\mathbf{r})\right|$ or $\left(1-\left|u_{z}(\mathbf{r})\right|\right)$ term.

The influence of imaging events (e.g., excitation, space encoding, signal sampling, etc.) on magnetization values is modeled by the Bloch equation [33]. We apply its discrete time solution in the form of rotation matrices and exponential scaling [34]. A similar approach has been successfully used in many advanced MRI simulators, e.g., SIMRI [59], ODIN [60], since it closely reproduces the physical processes of MRI.

In each cubic element, the influence of MRI events after successive time steps $\Delta t$ is expressed as follows:

$$
\begin{aligned}
\mathbf{A}_{\mathrm{MRI}}(\mathbf{r}, \Delta t) \\
\quad=\mathbf{E}_{\mathrm{RELAX}}(\mathbf{r}, \Delta t) \mathbf{R}_{z}\left(\Theta_{G}\right) \mathbf{R}_{z}\left(\Theta_{I H}\right) \mathbf{R}_{R F}(\mathbf{r}, \Delta t)
\end{aligned}
$$

where $\mathbf{E}_{\text {RELAX }}$ represents the relaxation phenomena, with spinlattice $(T 1)$ and spin-spin $(T 2)$ times. $\mathbf{R}_{z}$ is the rotation matrix about the $z$-axis used to model the influence of spatial encoding gradient $\mathbf{G}$ (rotation through angle $\Theta_{G}(\mathbf{r}, \Delta t)=\mathbf{G} \cdot \mathbf{r} \gamma \Delta t$ ) and magnetic field inhomogeneities $\Delta B$ (rotation through angle $\left.\Theta_{I H}(\mathbf{r}, \Delta t)=\gamma \Delta B(\mathbf{r}) \Delta t\right) \cdot \gamma$ is the gyromagnetic ratio and $\mathbf{R}_{R F}$ is the rotation matrix describing the influence of the radiofrequency $(\mathrm{RF})$ pulse and the slice selection gradient.

The MR signal coming from the imaged area in time $t$ is modeled as the sum of local transverse magnetizations over the entire image area

$$
\mathbf{S}(t)=\sum_{\mathbf{r}_{o} \in C} M_{x}\left(\mathbf{r}_{o}, t\right) \hat{\mathbf{i}}+\sum_{\mathbf{r}_{o} \in C} M_{y}\left(\mathbf{r}_{o}, t\right) \hat{\mathbf{j}}
$$

where $C$ is the collection of all cubic elements of the imaged area. The signal is sampled during an acquisition period and saved in the k-space matrix. Each subsequent excitation is performed with a different phase encoding step, and the acquired signals fill the successive matrix rows. The MR image is created by application of the fast Fourier transform (FFT) [61] to the fully filled matrix.

By default, the time step for modeling of MR flow imaging is equal to the time step from flow modeling. If needed (for quick trial calculations or time optimization issues), this time step can be changed. Moreover, it may be different during various stages of imaging, e.g., shorter during a slice selection and longer after a signal acquisition up to the time of the next excitation when there is less change in magnetization, which can lead to significant computational savings in simulation studies. However, this time step cannot be longer than the shortest time needed by all the fluid to pass from one grid node to another. In other case, it is possible that magnetization fractions can go (jump) more than one grid node in a single time step. A shorter time step, in turn, enables the time resolution to be increased, however, it can lead to an excessive increase of the simulation time. The model will usually apply a time step of the order of tens to hundreds of microseconds and a streaming distance (cubic element size) of the order of tens to hundreds of micrometers.

As regards the grid resolution, a restriction arises because of the need to have appropriate number of cubic elements in each image voxel. Due to the applied discrete-event solution of the Bloch equation, simulations are performed at discrete spatial locations and continuous distribution of spins has to be imitated. Thus, the number of cubic elements (also called isochromats) in each image voxel has to be chosen carefully to generate a smooth image intensity. First, to avoid image artifacts related to signal replication in the $\mathrm{k}$-space matrix, the cubic element size cannot be bigger than the image voxel size. Second, using insufficient number of cubic elements can lead to truncation artifact [60], in particular for abrupt image intensity changes. Moreover, previous studies have shown that, to model the intra-voxel phase variations [2], a sufficient number of cubic elements has to be assigned to each voxel [60]. On the other hand, too high number of cubic elements may result in spurious spin echo refocusing if not sufficient frequency spacing is provided [62] and in excessive simulation time.

In the model, the default value is set at 5 cubic elements per direction in each image voxel. It is based on previous studies 
[75], [60] as well as our own investigations to search well-compromised values in the context of image quality and simulation time [35]. In the case of more complex geometries (e.g., porous media), it is possible that more cubic elements per image voxel should be used.

In the model, the ideal spoiling of transverse magnetization is currently used. After each readout, the transverse magnetization of all cubic elements is set to zero. Thus, there is no possibility that any residual transverse magnetization can persist into the next repetition, even if short repetition time is needed. This simplification provides, however, numerical advantages to the simulation [18], e.g., there is no need to track the transverse magnetization after the readout up to the time of the next excitation, which can provide simulation time saving, especially when long repetition time is demanded.

In addition, the so-called hard-pulse approximation is used to represent different RF pulse shapes [72]. It allows the shaped RF pulses to be approximated by the sequence of short constant pulses of equal duration separated by periods of free precession, which perfectly suits for the applied iterative approach of MRI and flow simulations.

1) Parallel Algorithm of MR Flow Imaging: The imaged area is composed of cubic elements creating a rectangular box [see Fig. 3(a)]. One-dimensional (1-D) data decomposition (also called slice decomposition) [43] is used, i.e., the box is cut into equal-size slices. These slices are then assigned to processors. Since the MRI modeling involves magnetization changes that are based only on local data [see (3) and (7)], each processor performs this part of the algorithm independently. However, if magnetization transport takes place between cubic elements located in neighboring slices, there is a need to exchange data between these slices. For this purpose, the message passing paradigm is applied. To ensure efficient communication, the ghost vectors of cubic elements are used at slice interfaces. They are used to hold copies of data to be sent to neighboring slices as well as received from neighboring slices.

\section{RESULTS}

The proposed approach is verified in two steps. Firstly, the experimental validation is performed with a physical phantom consisting of three bifurcations. Then, we demonstrate the simulation possibilities of the model. Flow and MRI simulations in complex vascular structures are presented and inspected visually as well as quantitatively using flow characteristics curves.

All simulations and visualizations were performed by our in-house software. This software is built up of several modules that enable us to control all simulation stages, to look at virtual objects and simulated images, to monitor flow simulations as well as visualize the flow results.

\section{A. Comparison With In Vitro Experiments}

The computational model of MR flow imaging was validated both qualitatively and quantitatively in our previous paper [35] for different simple geometries (straight tube, U-bend tube, and single bifurcation) and for various flow conditions and parameters of MRI sequences. Both theoretical and experimental investigations were performed. In this paper, we

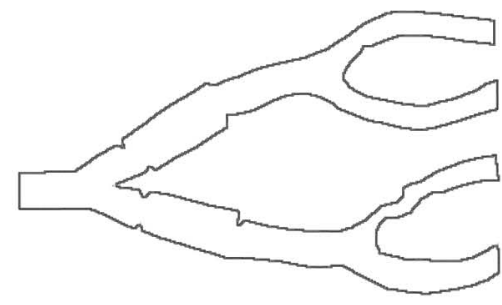

simulated phantom

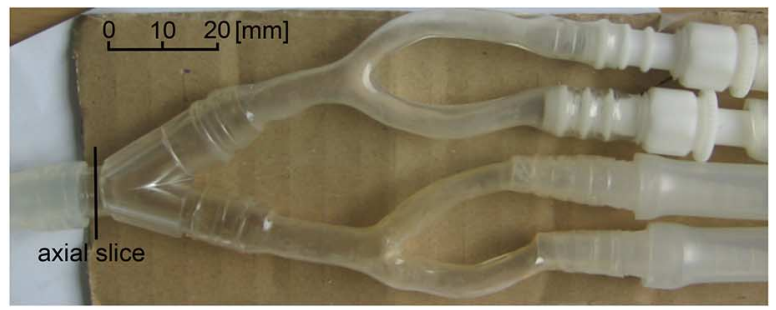

physical phantom

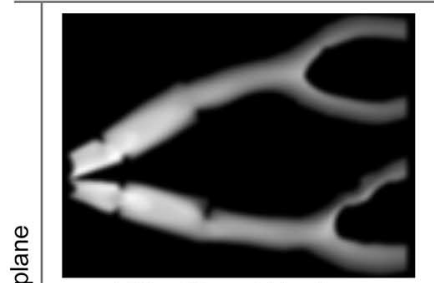

$\mathrm{TR}=50 \mathrm{~ms} \mathrm{TE}=5 \mathrm{~ms}$

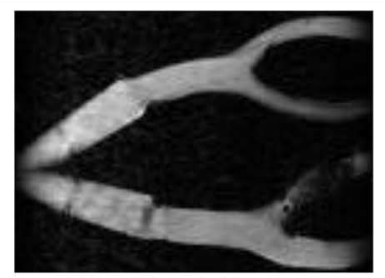

$\mathrm{TR}=50 \mathrm{~ms} \mathrm{TE}=5 \mathrm{~ms}$

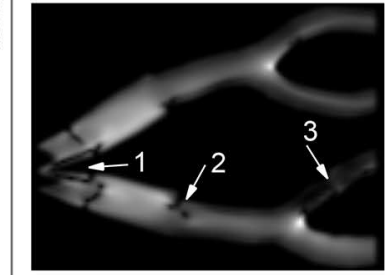

$\mathrm{TR}=50 \mathrm{~ms} \mathrm{TE}=20 \mathrm{~ms}$

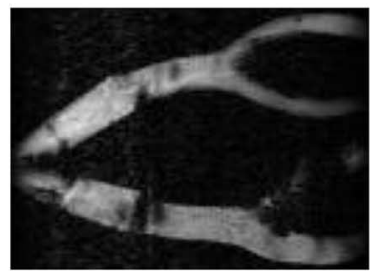

$\mathrm{TR}=50 \mathrm{~ms} \mathrm{TE}=20 \mathrm{~ms}$

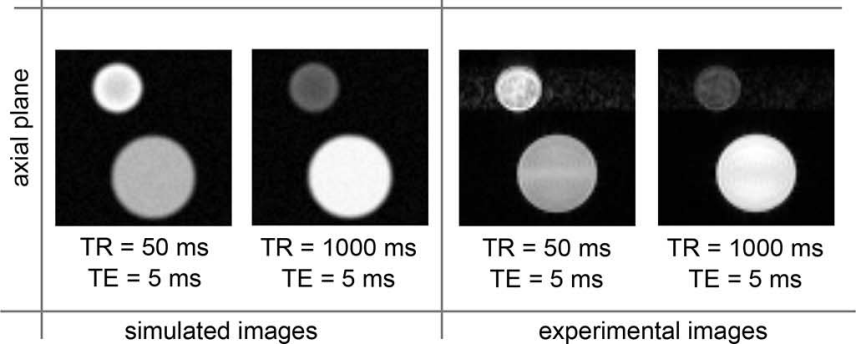

Fig. 6. Comparison between experimental and simulated GE images in comparable geometries. Images acquired in coronal and axial planes with different $\mathrm{TR} / \mathrm{TE}$ parameters. In the axial plane, a reference tube with static water can be also seen (bottom right of the image).

present experimental validation using a silicon phantom with three bifurcations (Fig. 6). Tubing radii range from 2 to $4 \mathrm{~mm}$. The tubes forming the first bifurcation (from the left side) are rigid and straight, while the second and third bifurcation shapes correspond to human aorta anatomy with an aneurysm (Elastrat phantoms, Geneva, Switzerland). The flow of cold tap water through the phantom was provided by a constant flow rate pump. MR imaging was performed on a Bruker Biospec 4.7T scanner (Bruker Biospin, Wissembourg, France) using the 2-D spoiled gradient echo sequence (FLASH). 
Using the proposed computational model, a similar geometry was constructed and MR imaging simulations were performed. Fluid behavior was modeled as the steady flow of a Newtonian liquid with literature-based properties for pure water at $10{ }^{\circ} \mathrm{C}: \mathrm{T} 1=2500 \mathrm{~ms}, \mathrm{~T} 2=2500 \mathrm{~ms}$, and kinematic viscosity $=1.3 \times 10^{-6} \mathrm{~m}^{2} / \mathrm{s}$ [74], [13]. To obtain comparable flow characteristics, constant velocity conditions were applied at the object inlet and outlets. At the inlet, the constant flow forced by the pump was used. At all outlets, we assumed that flow rate is the same, and flow velocities were calculated based on cross-sectional areas. Five cubic elements per direction were assigned to each voxel.

Fig. 6 shows some examples of experimental and corresponding simulated images. Images were acquired in the axial and sagittal planes with different parameters (TR/TE). A mean flow speed of $30 \mathrm{~cm} / \mathrm{s}$ was applied at the entrance of the phantom. Basic imaging parameters are reported on the images, while the others were set as follows: 1) sagittal images: flip angle $=30^{\circ}$, in-plane resolution $=0.625 \mathrm{~mm} \times 0.625 \mathrm{~mm}$, field of view $=90 \mathrm{~mm} \times 60 \mathrm{~mm}$ and slice thickness $=3 \mathrm{~mm}$, 2) axial images: flip angle $=40^{\circ}$, in-plane resolution $=0.5$ $\mathrm{mm} \times 0.5 \mathrm{~mm}$, field of view $=32 \mathrm{~mm} \times 32 \mathrm{~mm}$ and slice thickness $=4 \mathrm{~mm}$.

As far as images in the sagittal plane are concerned, at least two flow-related artifacts are clearly visible. First, the saturation of the longitudinal magnetization causes the signal to become weaker at increasing distance from the entrance. Second, the signal is reduced due to the intra-voxel phase dispersion [2] in the bifurcation neighborhood (first white arrow) and areas with complex shapes (second and third white arrows). The phase dispersion is probably the dominant reason of the signal dropout in the apex of the first bifurcation (first white arrow), like in previous studies [16]. In the areas with complex and irregular shapes (second and third white arrows), tubes are substantially narrowed. As a result, in these areas, strong velocity gradients can be observed, which can lead to phase dispersion and finally to signal diminution. When TE is increased, the period of time between excitation and signal acquisition also increases, and therefore the artifacts due to the intra-voxel phase dispersion are more pronounced.

The images in the axial plane (approximately perpendicular to the main flow direction, see photo of phantom in Fig. 6) were acquired using an additional silicon object with static water (reference object placed near the object of interest). This allows us to observe how the contrast of flowing versus static tissue can be regulated. When TR is short (i.e., $50 \mathrm{~ms}$ ) static water nuclei are saturated and give much less signal than flowing "fresh" water nuclei. In contrast, when TR is longer (i.e., $1000 \mathrm{~ms}$ ), static nuclei have more time for longitudinal relaxation and consequently give more signal than flowing nuclei.

A good agreement is observed between simulated and experimental images. However, there are some image features that show up the limitations of the model. For example, compared with simulated images, more prominent signal loss in the place where tubes are connected and higher noise can be observed in experimental images. On the other hand, simulated images appear to underestimate the signal close to walls. These differences can be ascribed to incompletely modeled imaging pro- cesses, e.g., approximated rectangular gradient waveforms or ideal receiver and gradient coils used in simulations. Other reasons can be found in the developed flow model. Due to computational efficiency issues, we applied the single-relaxation-time LBM scheme without any turbulence model (e.g., large-eddysimulations (LES) LBM model [76]). This LBM scheme could not resolve flow turbulences sufficiently with the used resolution and uniform grids [28]. Moreover, some differences in the MR images could also be explained by discrepancies between 2-D flow simulations and 3-D in vitro flow.

\section{B. Simulations in Complex Vascular Structures}

1) Vascular Structure Generation: The simulation process was started by generating a vascular system. The simulated vascularization of an adult liver was obtained by the use of the first model component (vascular network component) [Fig. 7(a)]. The initial vascular trees consisted of a few vessel segments whose geometry was chosen based on anatomical data [63]. The organ shape was bounded by the reconstructed liver geometry from $120 \mathrm{CT}$-scans of $1 \mathrm{~mm}$ thickness. The shape was filled in successive algorithm growth steps by new MFUs and, as a consequence, new vascular structures appeared. Detailed parameters concerning the initialization of the simulation as well as the physiological and functional parameters (e.g., blood flow and pressure at input/output, wall thickness ratio, size of MFU or probability of mitosis/necrosis) can be found in [21] and [70].

The simulated vascular system consisted of about 75000 vessel segments (about 25000 for each of the three hepatic vascular trees-arteries, portal veins, and hepatic veins) [Fig. 7(a)]. For further analysis (flow and MRI simulations), only the arterial tree was retained [Fig. 7(b)]. Moreover, vessel segments of diameter larger than $0.3 \mathrm{~mm}$ were taken into account [Fig. 7(c)]. In performance evaluation, we also considered more sophisticated/complex vascularizations.

2) Flow Simulations: Blood flow was simulated in the selected part of the arterial vascular tree (about 1200 vascular segments). Blood behavior was modeled as the steady flow of an incompressible Newtonian liquid with a kinematic viscosity of $3.4 \times 10^{-6} \mathrm{~m}^{2} / \mathrm{s}$. Constant pressure boundary conditions were applied at the entry and exit points (see Fig. 7(c) for sample values).

First, the analytical geometry was discretized with cubes of size $0.064 \times 0.064 \times 0.064 \mathrm{~mm}^{3}$. Next, cubic elements were found corresponding to walls, input and outputs, and boundary conditions were marked at these locations (see Section II-B). Finally, flow behavior was simulated by performing the collision and propagation steps in alternation until the desired convergence criterion was satisfied. The simulation was stopped when the global absolute difference of the velocity fields (with L2 norm) between successive iterations was less than $10 e^{-6}$.

Fig. 8 presents a visualization of the flow simulation results. The magnitude and direction of the flow are shown in a few cross-sections of each vessel segment. Fig. 8(a) shows all the selected arteries (about 1200), while Fig. 8(b) shows the sample parts of the arterial tree, at different levels ranging from several bifurcations to a single one. Curves of the obtained flow characteristics are presented in Fig. 9: pressure drop, volumetric flow rate drop, and velocity drop are shown in (a), (b), and (c), re- 


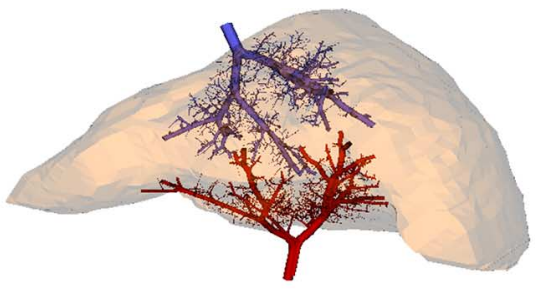

(a)

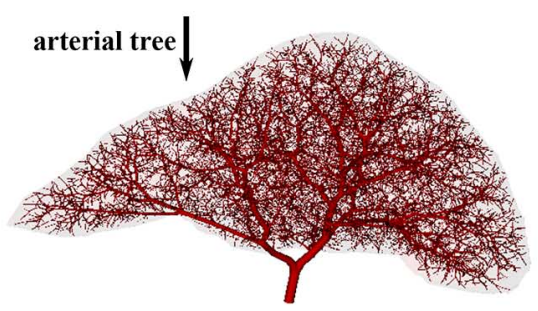

(b)

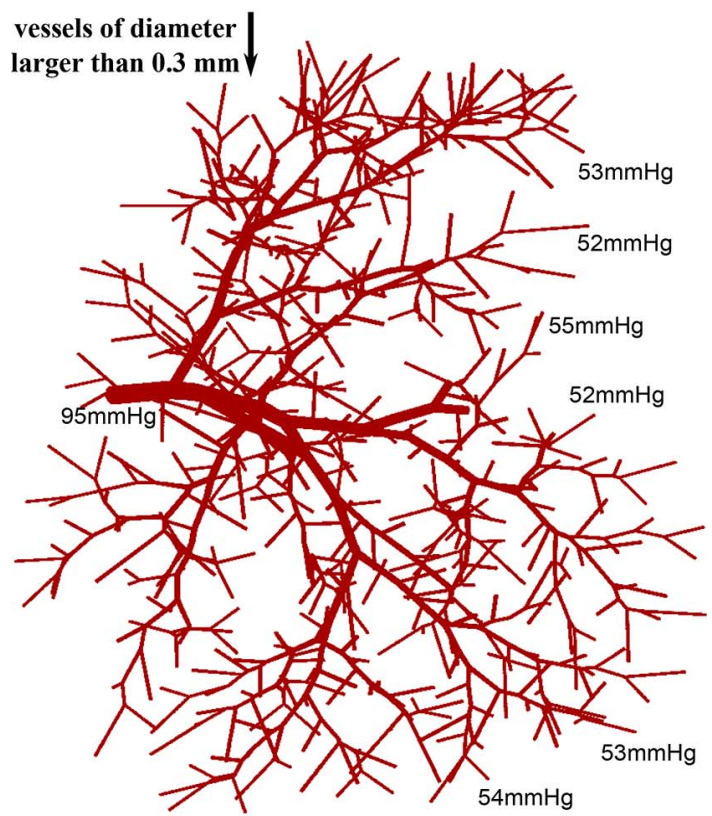

(c)

Fig. 7. Visualization of the vascular simulation results: (a) an adult liver with about 12000 MFUs and vessel segments (for the purpose of visualization, only the main hepatic arteries, portal veins and hepatic veins within liver shape are shown), (b) arterial tree, (c) arterial tree with vessel segments of diameter larger than $0.3 \mathrm{~mm}$. Sample values of blood pressure used for boundary conditions are also provided.

spectively. These characteristics are averaged for each vessel segment and presented along ten selected paths, from the root to terminal vessel segments. By comparing the obtained characteristics with previous works [69] and literature data [70], [71], it appears that similar patterns are observed: e.g., exponential drop of pressure in successive hepatic arteries, with the largest drop occurring in the most distant vessels, more linear velocity drop in successive bifurcations and high drop of volumetric flow in the larger arteries.

3) MR Flow Imaging Simulations: Based on the obtained flow characteristics and the proposed imaging model component, MR flow images were simulated. Two imaging sequences were used: 2-D spoiled gradient echo (GE) and 2-D spin echo
(SE) [72]. To mimic arterial blood, the relaxation times were set as follows: $\mathrm{T} 1=1000 \mathrm{~ms}$ and $\mathrm{T} 2=250 \mathrm{~ms}$ [73], [16].

Fig. 10 shows the simulated images in the axial plane from successive slices using the GE and SE sequences. We focused on a single bifurcation, which is marked by the green cuboid. The following imaging parameters were used: $\mathrm{TR}=50 / 1000$ $\mathrm{ms}$, TE $=5 / 20 \mathrm{~ms}$, in-plane resolution $=0.256 \mathrm{~mm} \times 0.256$ $\mathrm{mm}$, field of view $=11.7 \mathrm{~mm} \times 5 \mathrm{~mm}$, and slice thickness of $2.5 \mathrm{~mm}$. The simulated images suffer mainly from two flow-related effects: intra-voxel phase dispersion as well as inflow and mismatch between the $90^{\circ}$ and $180^{\circ}$ pulses. In the case of GE images, the signal dropout can be attributed mainly to the intravoxel phase dispersion which grows in the neighborhood of the bifurcation. The artifacts caused by the phase variations over a voxel are usually more pronounced when flow velocity grows or TE grows. For images in both the second row (after a TE decrease) and the third row (smaller blood flow and, consequently, smaller velocities), the signal diminution is reduced.

In the case of SE images, the flow-induced signal loss in stronger than in the GE images. SE images are similarly affected by the intra-voxel phase dispersion, however they can be additionally influenced by the mismatch between the $90^{\circ}$ and $180^{\circ}$ pulses. In the case of SE images, the signal intensity is significantly lower in the center of the vessel segments than near their walls. This is mainly due to the time-of-flight (TOF) effects [64] that usually cause vessels to appear as hyposignal areas or as totally black holes in the SE-based sequences (effect known as "flow voids" in clinical MRA) [65]. In detail, moving blood nuclei that are initially excited by the $90^{\circ}$ pulse may then exit the imaged slice without being rephased. As a consequence, these blood nuclei may not yield any signal. On the other hand, some nuclei not present in the slice during excitation may appear in this slice during rephasing but they also fail to yield any signal. The influence of this effect depends on the flow velocity, TE and slice thickness. For images in both the fifth row (after a TE decrease) and the sixth row (smaller blood flow and, consequently, smaller velocities), the signal diminution is reduced. Moreover, higher signal loss is observed in the vessel segment oblique to the image plane, probably because of the in-plane flow and significant intra-voxel phase variations [66]. The slower flow is generally visualized better.

Fig. 11 presents simulated images of a cross section through the arterial tree (as marked by the green cuboid) using both SE and GE sequences. To highlight the differences between flowing and static material, the area outside the vessels (i.e., cubic elements outside the vessels) also took part in the MRI simulation. For each cubic element outside the vessels, the flow was set to zero, while the relaxation times were set in the same way as used for the blood. Here, different combinations of contrast parameters (TR/TE) were tested, while other imaging parameters were set as follows: in-plane resolution $=0.32 \mathrm{~mm} \times 0.32 \mathrm{~mm}$, field of view $=50.56 \mathrm{~mm} \times 44.16 \mathrm{~mm}$, and slice thickness of $5 \mathrm{~mm}$. The peak velocity in the imaged vessel segments was about $50 \mathrm{~cm} / \mathrm{s}$.

The presented images (Fig. 11) show primarily the differences in longitudinal magnetization recovery. In images where TR is small enough (e.g., $50,200 \mathrm{~ms}$ ), the static material is saturated since there is insufficient time between successive excita- 


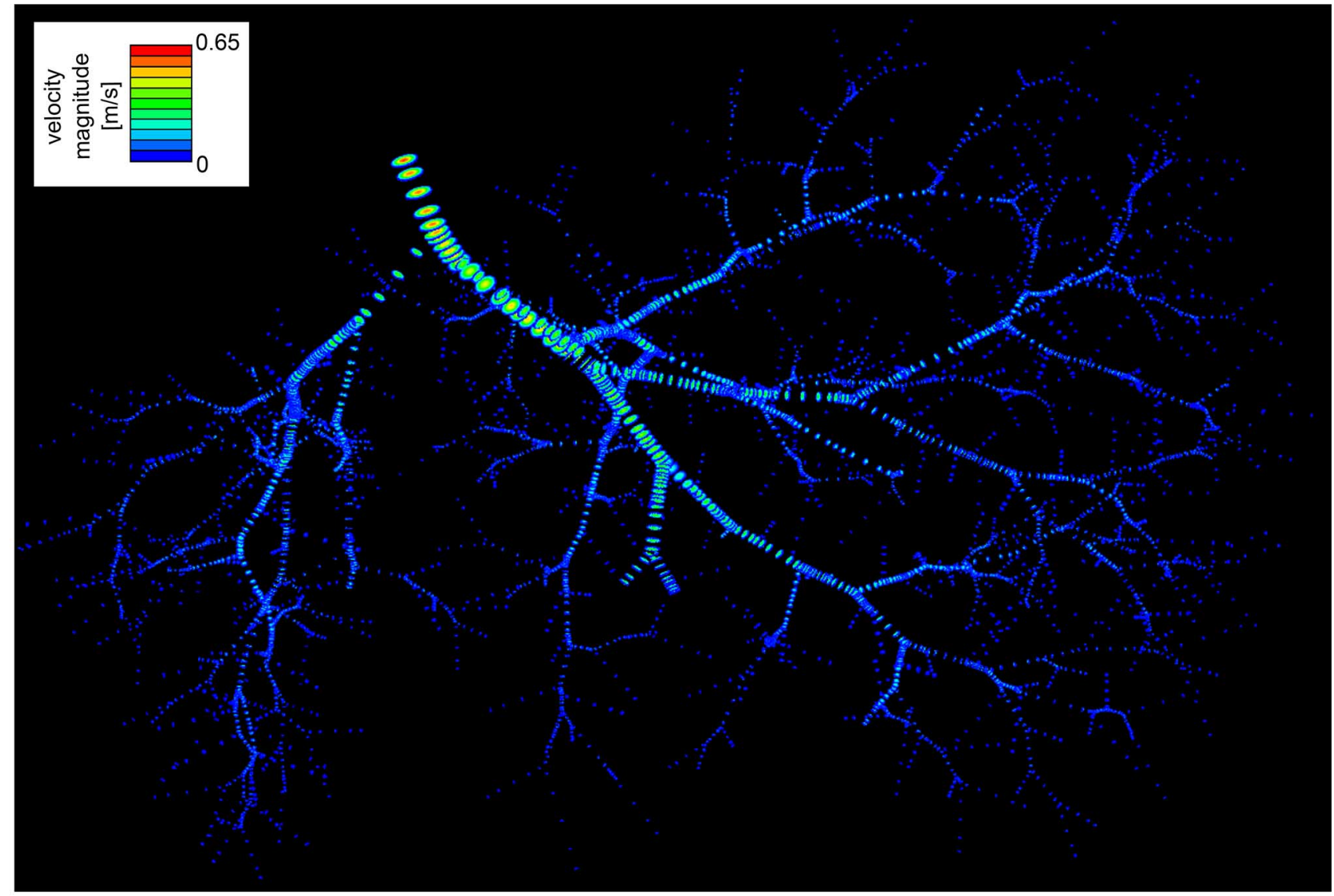

(a)
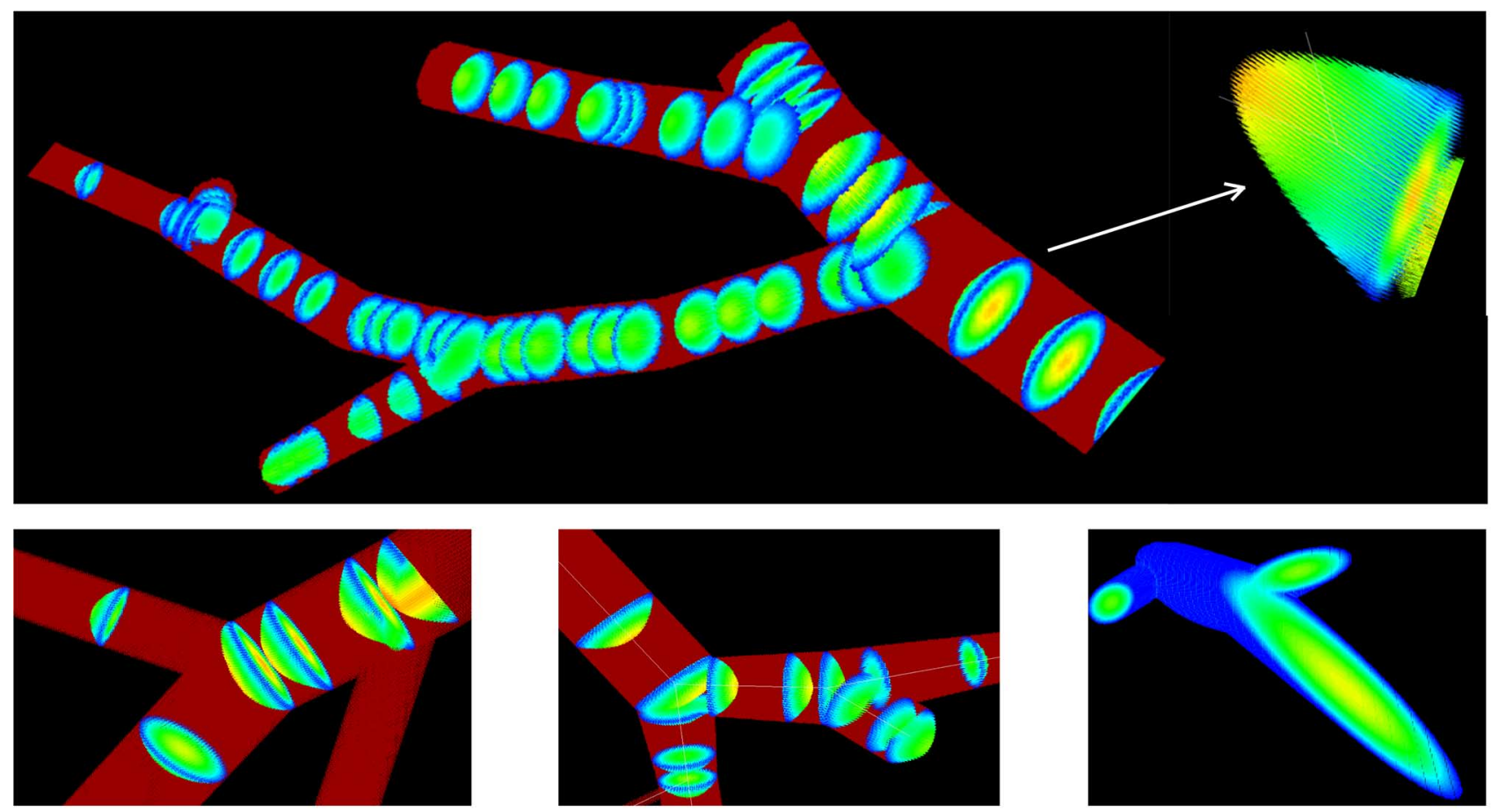

(b)

Fig. 8. Flow simulation results; visualization at different levels: (a) all the selected arteries, (b) parts of the arterial tree. Colors and lines in a few cross-sections of each vessel segment represent velocity magnitude and flow direction. 


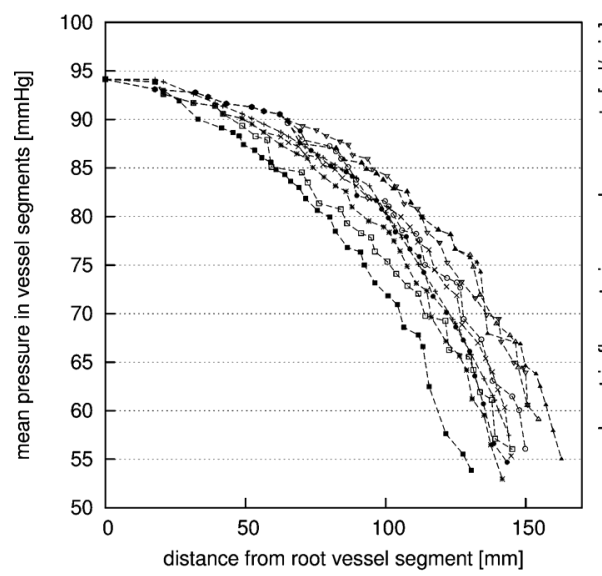

(a)

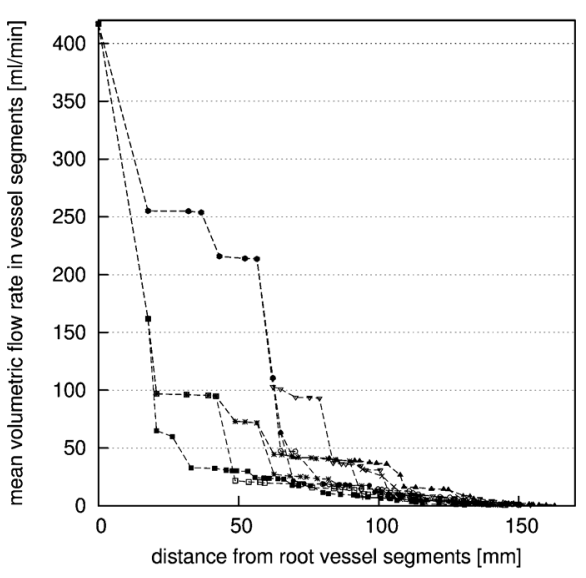

(b)

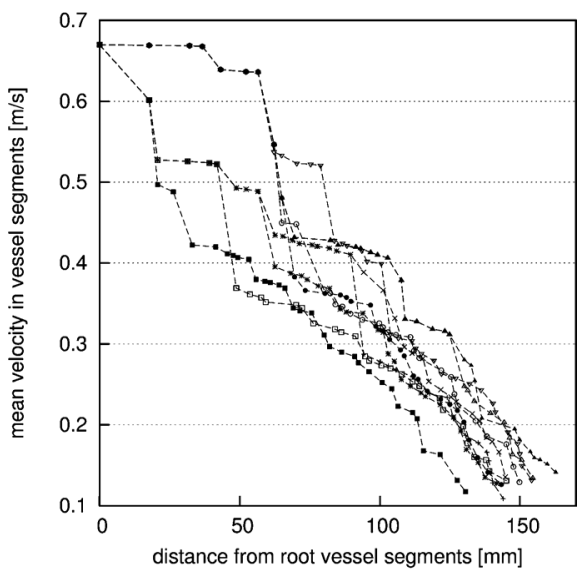

(c)

Fig. 9. Flow simulation results; curves showing flow characteristics averaged in vessel segments: (a) pressure drop along vessel segments passing from the root to terminal vessel segments (ten selected paths), (b) flow rate drop along vessel segments passing from the root to terminal vessel segments (ten selected paths), (c) velocity along vessel segments passing from the root to terminal vessel segments (ten selected paths).

tions to recover its longitudinal magnetization. As far as blood is concerned, "fresh" unmagnetized nuclei (with complete/full longitudinal magnetization) flow continuously into the image slice. The blood velocity determines how fast they replace the saturated flowing nuclei. These "fresh" nuclei are able to give more transverse magnetization in the next RF excitation, and consequently more signal. Thus, vessels appear brighter than their static surroundings. When TR is much longer, there is sufficient time for complete recovery of longitudinal magnetization of static material. Then, other flow-induced effects dominate which cause vessels to appear darker.

There are at least a few reasons why vessels appear black in SE images. First of all, signal loss can be caused by the intra-voxel phase dispersion in the absence of flow compensation methods. We performed additional simulations to investigate the difference between the transverse magnetization values for flowing and static material with different flow velocities using single excitation. Fig. 12 presents the amplitude and phase of the signal measured for a single cubic element placed along the center line of a straight tube in the isocenter and just next to the isocenter of the magnetic field. The tube length was 0.4 $\mathrm{m}$, so no fresh nuclei appeared in the observed cubic element. Sequence parameters: $\mathrm{SE}, \mathrm{TE}=1.5 \mathrm{~ms}$, acquisition time $=1$ $\mathrm{ms}$, sinc-shaped RF pulse time $=0.3 \mathrm{~ms}$. First, we can see that the amplitude of the signal at time TE is lower with flow than it is without flow. Second, the phase for different flow velocities shows different values at time TE. We can also observe that the phase accumulated due to the blood flow is directly proportional to the blood velocity, which is in accordance with previous studies [2]. Therefore, if the signal in a voxel comes from cubic elements with different flow velocities, the intra-voxel phase dispersion can lead to signal diminution. As a result, the signal in such a voxel is weaker than it would be without flow. These observations can explain the signal dropout for vessels when there is no flow compensation method. Moreover, SE images can be influenced by mismatch between the $90^{\circ}$ and $180^{\circ}$ pulses. However, in the presented images, this flow-induced effect seems to be small since most of the vessels in the chosen slice do not cut it orthogonally. In addition, some of the vessel segments visible in SE images are not present in GE images (see black arrows in Fig. 11). It can be attributed to the saturation of blood nuclei due to their long dwell time in the slice because of small blood velocity and/or long distance from the entrance of "fresh" nuclei. The presented example shows how blood may be imaged as low or high in signal with respect to the stationary tissue.

In the presented images, the high and low image intensities as well as the flow-induced image disturbances are consistent with prior theoretical and experimental studies on MR flow imaging, e.g., signal dropout in images in Fig. 10 due to the washout of excited spin [9], [64], [17], signal diminution in images in Fig. 11 due to the IVPD artifact [2], or saturation effects in images in Fig. 11 [16].

\section{Evaluation of Computational Performance}

All components of the model were implemented in the $\mathrm{C}++$ programming language. Algorithms were parallelized with the support of the message passing interface (MPI) [44] (communication between distributed-memory machines), and OpenMP Application Programming Interface [45] (communication within shared-memory). The Intel $\mathrm{C}++$ Compiler 10.1 [67] and MVAPICH2 ver. 1.8.1 [68] (MPI2 implementation over Infiniband networks) were used. The hybrid parallelization of the first model component was supported by mixed MPI+OpenMP. With respect to the second and third model components, one-level parallelism was applied using MPI. For performance evaluation, we made use of the Multi-Processing Environment (MPE) library with the graphical visualization tool Jumpshot-4 [44].

Simulations were performed on a cluster of sixteen SMP servers running Linux 2.6 and connected by an Infiniband network. Each server was equipped with two quad-core CPUs (64-bit Xeon 2.66 GHz CPUs) with $2 \mathrm{MB}$ L2 cache, $16 \mathrm{~GB}$ of RAM and Infiniband $10 \mathrm{~GB} / \mathrm{s} \mathrm{HCA}$ connected to a PCI-Express port. Moreover, the solution was also checked on a personal 

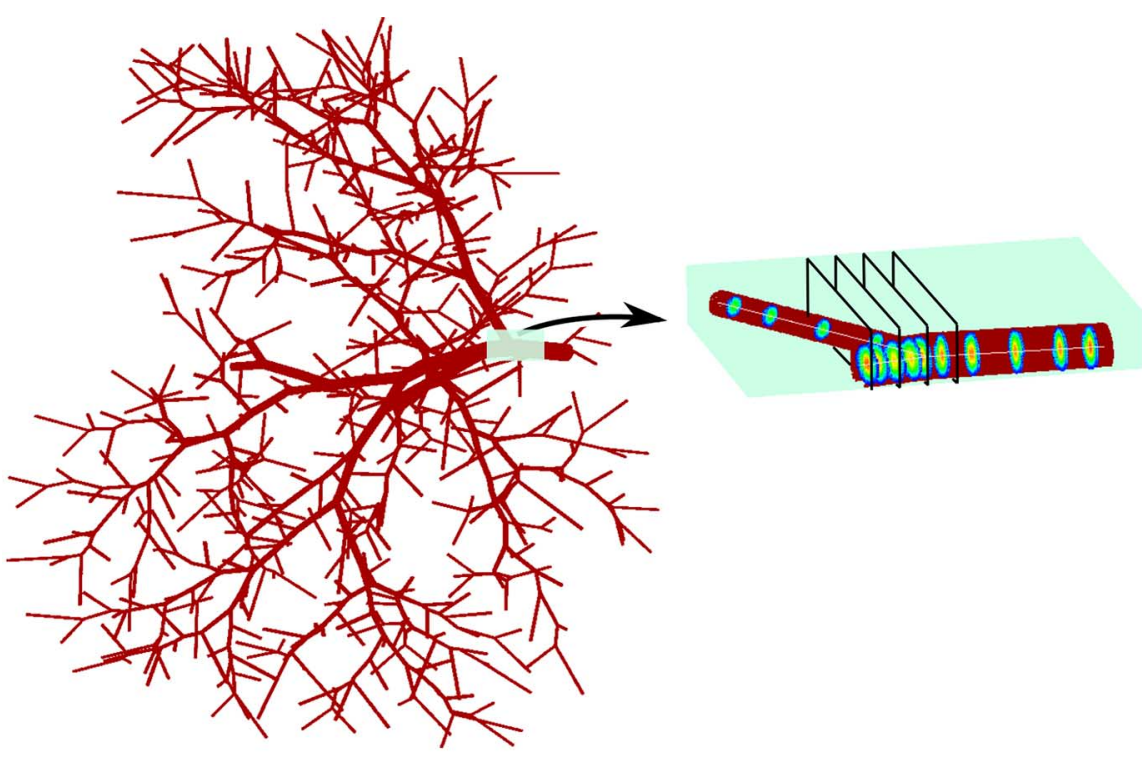

(a)

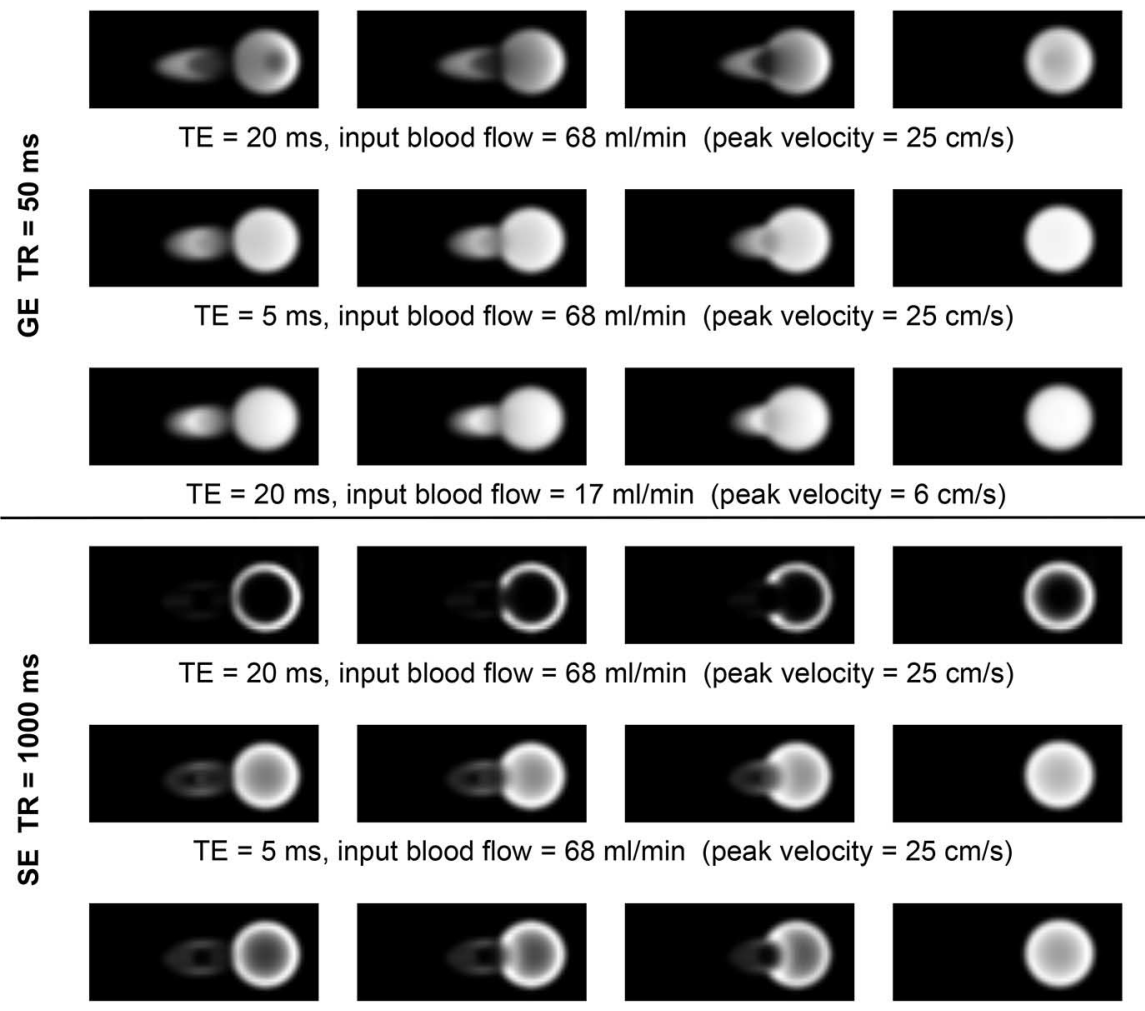

$\mathrm{TE}=20 \mathrm{~ms}$, input blood flow $=17 \mathrm{ml} / \mathrm{min}$ (peak velocity $=6 \mathrm{~cm} / \mathrm{s}$ )

(b)

Fig. 10. Simulated MR images acquired by SE and GE sequences in the axial plane: (a) green cuboid indicates a part of the arterial tree that is shown in the images, (b) series of images for different imaging and flow parameters.

computer equipped with Intel Core i7 CPU $(1.73 \mathrm{GHz})$ and 8 GB of RAM under MS Windows 7 in MS Visual Studio 2010.

Two metrics were used for qualitative analysis of the performance: speedup and efficiency. ${ }^{1}$ Moreover, we used the fundamental formula, known as Amdahl's law [77]. Briefly, this

\footnotetext{
${ }^{1}$ Speedup is the time necessary to solve a problem sequentially divided by the time required by its parallel version (i.e., how much a parallel algorithm is faster than its sequential version) [43]. Efficiency is defined as the speedup divided by the number of processors.
}

law states that the maximum speedup (Amdahl's upper bound) is limited by the fraction of time needed to perform nonparallelizable code components, no matter how many processors are used.

The computational performance of the vascular model component was tested for different sizes of vascular networks, ranging from small-size (about $100 \mathrm{MFUs)}$ to large-size configurations (about 50000 MFUs and consequently about 300000 


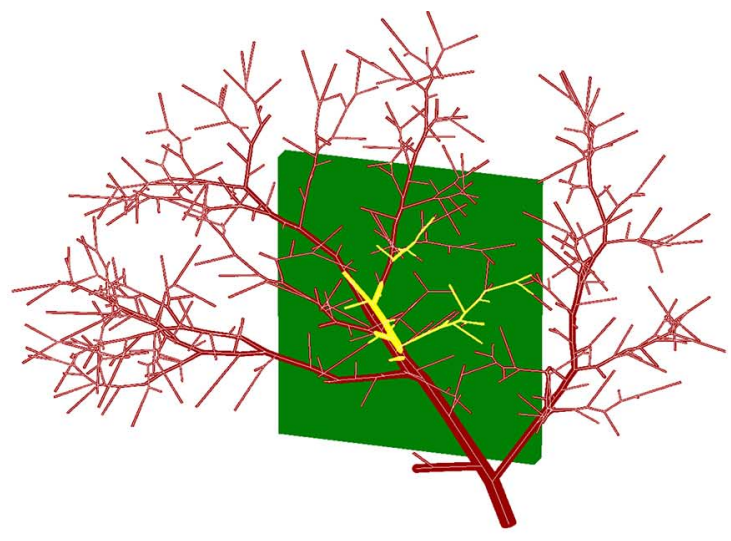

(a)

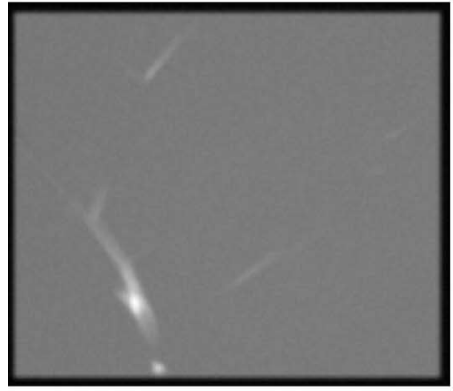

$\mathrm{GE}$ TR $=50 \mathrm{~ms} \mathrm{TE}=10 \mathrm{~ms} \mathrm{FA}=30^{\circ}$

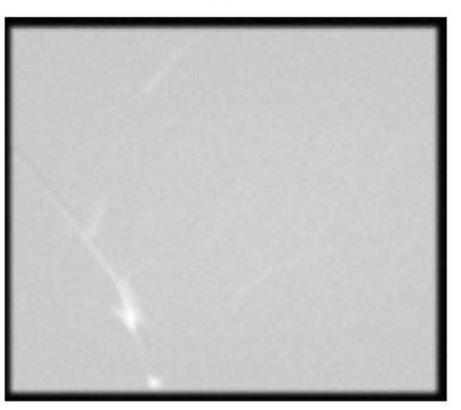

$\mathrm{GE} T \mathrm{TR}=200 \mathrm{~ms} \mathrm{TE}=10 \mathrm{~ms} \mathrm{FA}=30^{\circ}$

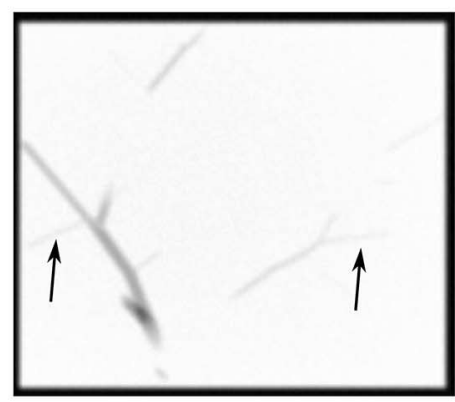

SE $T R=2000 \mathrm{~ms}$ TE $=10 \mathrm{~ms}$

(b)

Fig. 11. Simulated SE and GE images of the slice through the entire volume containing the arterial tree: (a) green cuboid indicates an excited slice, (b) simulated images with different sequences and with different TR/TE parameters.

vessel segments). The obtained mean speedups are reported in Table I. It can be seen that the parallelization provides a substantial speedup. However, the efficiency is far from $100 \%$. This can be explained by the upper bound speedup resulting from the Amdahl's law. The nonparallelizable code consumes about $3 \%$ of the total CPU time needed for serial execution of the whole algorithm. As a consequence, the upper bound speedup is equal to about 26 with 128 processors, while 33 is the maximum speedup when the number of processors tends to infinity. In practice, the obtained acceleration allows us to simulate the vascular system with 50000 MFUs in less than $1 \mathrm{~h}$ with 128 processors (instead of $21 \mathrm{~h}$ with a single processor machine).

In analysing the performance of flow simulations, different sizes of vascular trees were taken into account (from 10 to 4000 vessel segments). Table I shows the obtained mean speedups. We see that the parallelization can significantly reduce the simulation time. On the one hand, it offers the possibility of studying more complex vascular networks, which can be useful in cases of tumoral hypervascularization. On the other hand, multiple test simulations in simple vessel configurations can be performed in a reasonable period of time. The flow results presented here (see Fig. 8) were obtained for about 1200 vessel segments and were simulated using 128 processors in approximately $12 \mathrm{~h}$ (instead of 14 days with a single processor machine).

The parallelization of the MR flow imaging algorithm was tested for 2-D and 3-D objects, with different sizes of images (from $64 \times 64$ to $4096 \times 4096$ ) and various combinations of TR and TE parameters. In Table I, we report the obtained mean speedups. The acceleration is significant. A high efficiency is also observed, e.g., $80 \%-90 \%$ for $4,8,16,32$ processors. Owing to this parallelization, the simulation of each MR flow image presented in Fig. 11(b) $(795 \times 690 \times 70$ cubic elements and $159 \times 138$ image voxels) took an average about $3 \mathrm{~h}$ with 128 processors instead of eight days using one processor. The simulation of each MR image in Fig. 10(b) took about 4 min using 128 processors.

\section{DISCUSSION AND SUMMARY}

We present a new computational model for MR flow imaging. The model consists of three components: 1) vascular structure generator, 2) flow solver, and 3) MRI simulator. The coupling of these three components together in a single solution allows us to track (back and forward) and control a wide range of physical processes involved in flow image formation. During in vivo as well as in vitro experiments, such a detailed control is usually impossible or hardly accessible. In the proposed model, we are able to control physiological processes influencing vascular geometries, hemodynamic parameters, and MR imaging settings. Such a three-component solution brings the possibility to explain data, observations and hypotheses based on collaborative knowledge which may be more detailed than if provided by studies using the individual modeling components.

Such an approach to MRI modeling has not been proposed before. To date, there were many computational studies consid- 


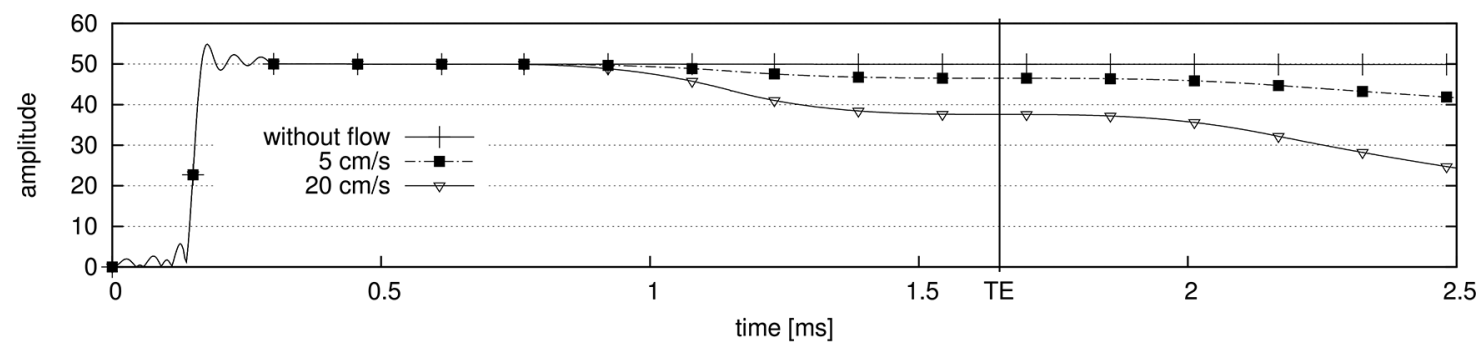

(a)

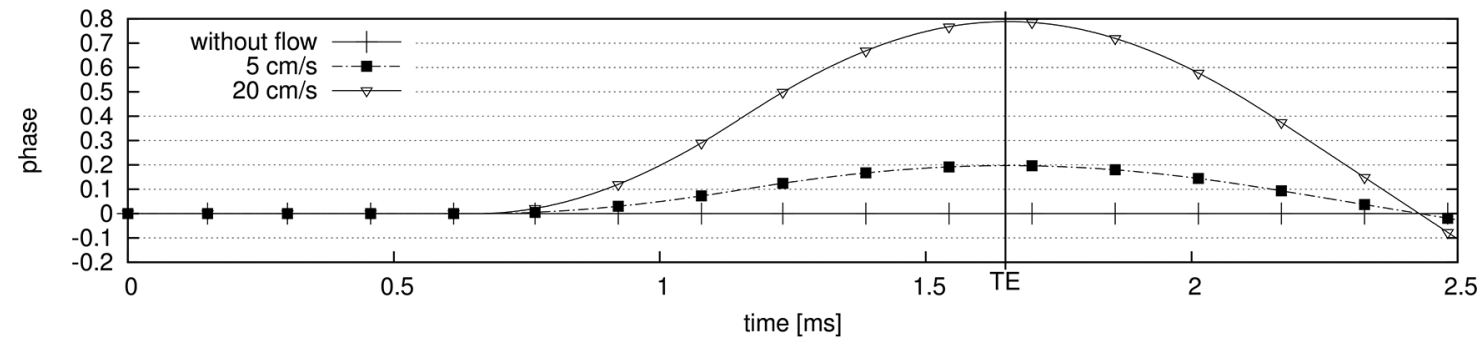

(b)

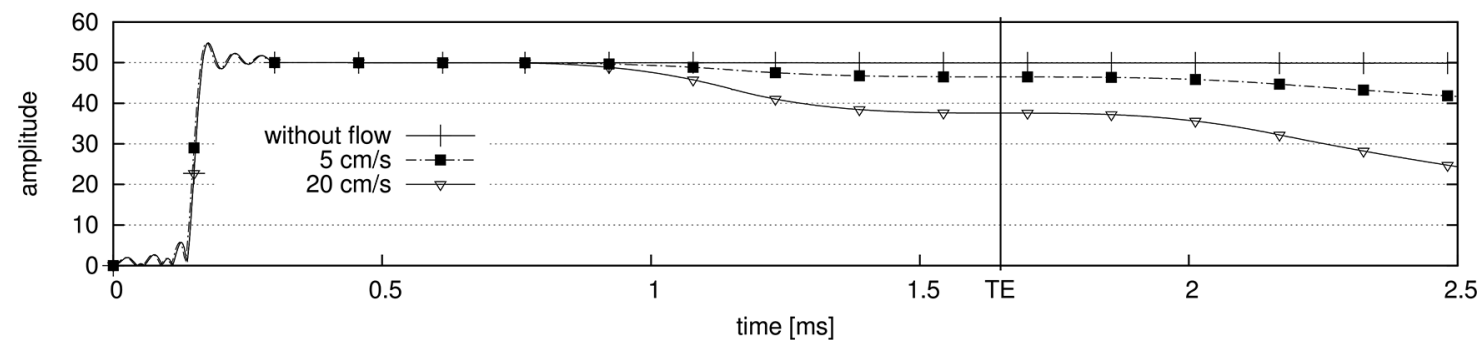

(c)

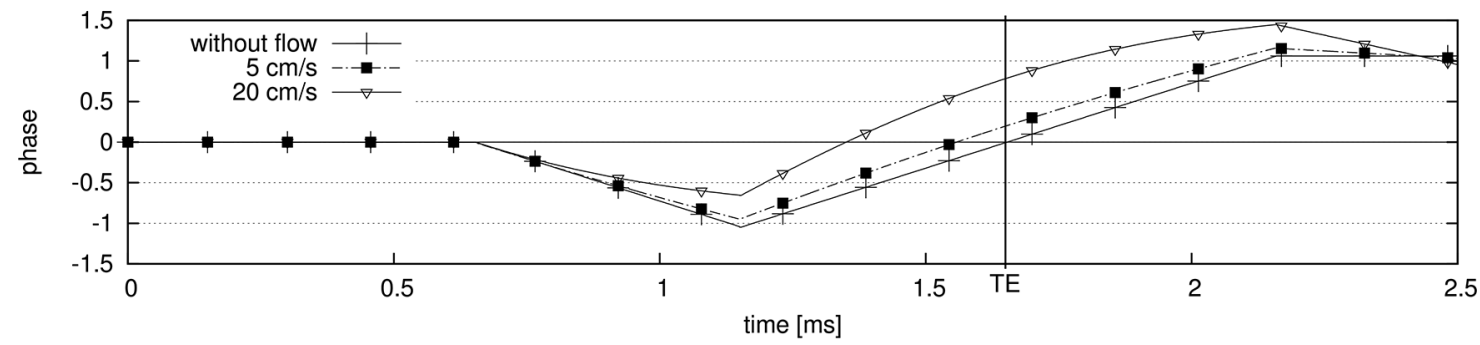

(d)

Fig. 12. Illustration of the influence of flow on (a), (c) amplitude and (b), (d) phase of the transverse magnetization in a single cubic element. Single cubic element is placed (a), (b) in the isocenter and (c), (d) just next to the isocenter of the magnetic field. Two values of flow (5 cm/s, $20 \mathrm{~cm} / \mathrm{s})$ and no flow are considered.

TABLE I

Computational Performance of the Three Model Components. Mean SPEedup (S) AND EFFiciency (E) VAlues Versus Number of Processors

\begin{tabular}{c|cc|cc|cc}
\hline number of & \multicolumn{2}{|c|}{ vascular generation } & \multicolumn{2}{|c|}{ flow } & \multicolumn{2}{c}{ MR flow imaging } \\
processors & $\mathrm{S}$ & $\mathrm{E}$ & $\mathrm{S}$ & $\mathrm{E}$ & $\mathrm{S}$ & $\mathrm{E}$ \\
\hline \hline 2 & 1.8 & $90 \%$ & 1.7 & $85 \%$ & 1.7 & $85 \%$ \\
4 & 3.5 & $87 \%$ & 3.0 & $75 \%$ & 3.1 & $78 \%$ \\
8 & 4.0 & $50 \%$ & 5.4 & $68 \%$ & 6.8 & $85 \%$ \\
16 & 7.0 & $44 \%$ & 10.2 & $64 \%$ & 13.4 & $84 \%$ \\
32 & 12.0 & $38 \%$ & 18 & $56 \%$ & 27.0 & $85 \%$ \\
64 & 17.0 & $27 \%$ & 31 & $48 \%$ & 43.0 & $67 \%$ \\
128 & 21.2 & $17 \%$ & 47 & $37 \%$ & 75.5 & $60 \%$ \\
\hline
\end{tabular}

ering vascular structures generation, flow simulations, or MR flow imaging separately. Classically, flow behavior in large vascular trees was simulated with the use of computationally inexpensive 1-D models [78], [79], [80]. Although these models are able to yield fast results that are satisfactory for a preliminary insight (e.g., direction of mean flow) [32], they are not sufficient to provide data for detailed MR simulations. For instance, they do not take into account the bifurcation geometry (e.g., bifurcation angle) and the flow profile is assumed to be the same at any point in the vessel.

There have also been many studies on flow modeling in 3-D vascular structures. However, these studies were usually limited to small-size domains [80], e.g., to a single vessel [81] or bifurcation [19], [82]. Recently, with the substantial advance in design of parallel computers, a computational solution has been proposed for high-resolution flow in large 3-D vascular networks [31]. Tens or hundreds of thousands of parallel processors have been used to solve the Navier-Stokes flow equations by a high-order spectral element spatial discretization [83] in the complex human brain arterial tree. The flow simulations 
took a few days for about one hundred vessels. The most recent computational paradigm in flow modeling couples multi-scale simulations (atomistic, at the level of red blood cells, and continuum, i.e., large arteries) by means of enormous computer resources: IBM Blue Gene/P supercomputer (131 072 processors and 5TB of data) [84] or massive GPU clusters (4000 GPUs) [85]. Although all these solutions represent a major advance in CFD, they are still hard to use in frequent simulations since they require access to huge computational and memory resources. Moreover, the procedure of simulation preparation is also timeconsuming and can change according to different geometries and/or initial conditions.

By contrast, the proposed LBM-based flow model was able to provide 3-D high-resolution results for hundreds and thousands of vessels in a relatively short time, i.e., in about $12 \mathrm{~h}$ on a middle-class computer cluster with only 128 processors (32 quad-core CPUs). Compared to the numerical Navier-Stokes equation solvers, there was no need for an explicit solution of pressure dynamics. Grids for complex geometries were generated in a fully automatic way and boundary conditions were straightforward to apply. There was also no need to use massive computational resources. Clearly, our approach has some limitations compared to Navier-Stokes based solvers, due to features inherited from LBM (e.g., the uniform mesh) or from the applied LBM model (e.g., steady flow behavior). However, the numerous advantages listed above, as well as many other strong points [28], cause that the flow model component provides a computationally efficient way for testing many scenarios in combination with the MR flow imaging simulator.

We compared the results obtained by the applied LBM algorithm with a numerical solution of the Navier-Stokes equations. The OpenFoam software [89] was used to simulate flow with the use of the Navier-Stokes equations. We used the icoFoam solver which is the transient solver for incompressible, laminar flow of Newtonian fluids. It solves the incompressible laminar Navier-Stokes equations using the PISO algorithm. Two geometries were verified: a 2-D and 3-D single-vessel segment as well as a 2-D single bifurcation (i.e., three vessel segments). In both cases, no significant differences were observed in the flow characteristics obtained from the two solvers. However, the LBM solver yielded results in a much shorter time than the numerical solution of the Navier-Stokes equations. For instance, for the 3-D vessel segment (length $17 \mathrm{~mm}$, radius $1.7 \mathrm{~mm}$, grid resolution $0.064 \mathrm{~mm} \times 0.064 \mathrm{~mm} \times 0.064 \mathrm{~mm}$ ), the simulation time using one CPU and LBM was about $5 \mathrm{~h}$, as against about 20 $\mathrm{h}$ using the numerical solution of the Navier-Stokes equations. Therefore, we limited this comparison to three vessel segments.

Several other solutions have also been proposed for the computational modeling of MR flow imaging. They can be divided into two groups: Lagrangian- (e.g., [13], [18]) or Eulerian-based (e.g., [14], [16]). Lagrangian-based solutions use flow pathlines to track magnetized particles and change the magnetic conditions influencing them. While this approach is physically intuitive, it is known to be computationally expensive and not so efficient in complex geometries where particle tracking can fail. Moreover, the algorithms for compensation of regions of low particles density can be needed since the densities of particles are not uniform over space [18], while in real fluids they are. On the other hand, the most advanced Eulerian-based solutions [14], [16] use Bloch equation integration with an additional magnetization transport term in 2-D objects. This computational approach eliminates the necessity to track magnetic particles along pathlines during imaging, and therefore is able to speed up calculations. However, it can hinder the modeling of the spatial-temporal interaction of flow with MRI events. Therefore, an additional geometrical procedures had to be developed to take into account the displacement artifact [10], e.g., mesh transformation for mapping the physical plane into the MR image plane [16]. Other gradient arrangements, different RF pulses or 3-D imaging may require additional algorithms or some improvements in the current algorithms to take account of the misregistration effects. Besides, all the previous works dealt with simple vascular geometries having a single bifurcation at most.

The advantage of our approach stems from coupling the magnetization transport algorithm (2-D/3-D analytical operations) with the discrete-time solution of the Bloch equation. Hence, the Eulerian coordinate approach is retained and, at the same time, flow-related artifacts are automatically taken into account. No additional geometrical procedures, such as mesh transformation [16], are needed. Consequently, modifications in imaging sequence events (e.g., additional RF pulses, magnetic gradients) only require changes in the arrangement of MRI events in time and/or their parameters. Another advantage of the approach is its computational efficiency, which allows us to consider complex vascular geometries (with thousands and more vessel segments). Since such geometries require the use of lattices with high spatial resolution compared to image resolution, parallel computing appears very useful for adequate tracking of magnetization transport.

Parallel computing could also appear very useful when a simulation time step has to be small enough. In the proposed approach, magnetization can be directly (i.e., in a single time step $\Delta t$ ) transferred only between neighboring grid nodes. As a result, the time step should be chosen carefully. We cannot choose too long time steps since then it is possible that for fast-flowing fluid the magnetization portions will jump over grid nodes. On the other hand, unreasonable reduction of time step can lead to excessive simulation time. In addition, if we even choose a time step providing the transfer only between neighboring grid nodes, differences between real and simulated images may appear. We experimentally verified that these differences usually decrease with the decrease of the time step. Shorter time steps could be particularly preferable for complicated geometries, and/or complex flow patterns.

Our future investigations will deal with more complex vascular networks. First, we plan to use the whole generated arterial tree, and then the two/three connected vascular trees (e.g., arteries, veins, and portal veins). This will lead to the possibility of simulating MR images of whole organs or more extensive regions, e.g., abdomen, by 3-D MR angiography sequences. It is noteworthy that geometric sensitivity studies have shown that it is better to examine vessel-shape anomalies as part of a more extensive vascularization than as andependent small sector [86]. We also plan to extend the model by contrast agent propagation and pharmaceutical product distribution components. 
The superiority of LBM over Navier-Stokes solvers in mixtures (multi-component fluids) flows has been proven in many studies [87], [88], [55].

Ongoing research also includes issues concerning other types of flow and blood rheology. In the proposed model, the full history of magnetization evaluation can be tracked and changes or even replacement of flow maps during imaging do not require any algorithm modifications. Thus, there is no restriction to incorporate turbulent or pulsatile flows. As far as the parallelization is concerned, some improvements can be applied to flow simulations. The obtained mean speedups are far from linear compared with the parallelizations used in other works [57], [58]. In our case, we aimed to find a trade-off between memory consumption and data organization in memory (which influenced efficiency). The considered vascular network required the use of large number of lattice nodes which filled the rectangular box sparsely (i.e., there were many nodes outside vessels). For this reason, we concentrated more on memory consumption, thus allowing the study of more vessels. We applied a 1-D list of fluid nodes instead of the full 3-D table, which consequently involved indirect addressing. Although such data representation reduced memory consumption, at the same time, it limited the reusage of cache memory. Despite this, we envisage that more work on parallel computing in the model will lead to better computational efficiency. Moreover, grid-refinement techniques [90] can help to reduce memory consumption.

In conclusion, we show the technical possibilities and potential of a three-component model for MR flow imaging. The model aims at explaining and exploring the formation and appearance of MR images using the collaborative knowledge obtained from many processes and systems, based on vascular geometry, flow patterns and imaging technology. Application of the model in some specific medical applications (e.g., searching markers of hepatocellular carcinoma or hepatic stenosis after liver transplantation [7]) may clarify the true dynamics and collaboration of the processes involved. The broad possibilities of this approach provide motivation for ongoing attempts to develop the model further.

\section{ACKNOWLEDGMENT}

We authors would like to thank Dr. W. Kwedlo for the excellent hardware support with the computing cluster at Bialystok University of Technology and also Dr. J.-J. Bellanger for his interest in numerical aspects of this work. Dr. M. S. N. Carpenter post-edited the English style.

\section{REFERENCES}

[1] Modeling and Simulation in the Medical and Health Sciences, J. A. Sokolowski and C. M. Banks, Eds.. Hoboken, NJ: Wiley, 2011.

[2] D. G. Nishimura, A. Macovski, and J. M. Pauly, "Magnetic resonance angiography," IEEE Trans. Med. Imag., vol. 5, no. 3, pp. 140-151, 1986.

[3] M. Mescam, M. Kretowski, and J. Bezy-Wendling, "Multiscale model of liver DCE-MRI towards a better understanding of tumor complexity," IEEE Trans. Med. Imag., vol. 29, no. 3, pp. 699-707, Mar. 2010.
[4] J. C. Lasheras, "The biomechanics of arterial aneurysms," Annu. Rev. Fluid Mechan., vol. 39, no. 1, pp. 293-319, Jan. 2007.

[5] A. L. Sverdlov, D. T. Ngo, M. J. Chapman, O. A. Ali, Y. Y. Chirkov, and J. D. Horowitz, "Pathogenesis of aortic stenosis: Not just a matter of wear," Am. J. Cardiovas. Disease, vol. 1, no. 2, pp. 185-199, Jul. 2011.

[6] R. W. J. Ryan, A. Chowdhary, and G. W. Britz, "Hemorrhage and risk of further hemorrhagic strokes following cerebral revascularization in Moyamoya disease: A review of the literature," Surg. Neurol. Int., vol. 3, no. 72, Jun. 2012.

[7] A. H. Caiado, R. Blasbalg, A. S. Marcelino, M. da Cunha Pinho, M. C. Chammas, C. da Costa Leite, G. G. Cerri, A. C. de Oliveira, T. Bacchella, and M. C. Machado, "Complications of liver transplantation: Multimodality imaging approach," Radiographics, vol. 27, no. 5, pp. 1401-1417, Sep. 2007.

[8] K. E. Wood, "Major pulmonary embolism: Review of a pathophysiologic approach to the golden hour of hemodynamically significant pulmonary embolism," Chest, vol. 121, no. 3, pp. 877-905, Mar. 2002.

[9] L. Axel, "Blood flow effects in magnetic resonance imaging," Am. J. Roentgenol., vol. 143, no. 6, pp. 1157-1166, Dec. 1984.

[10] D. G. Nishimura, J. L. Jackson, and J. M. Pauly, "On the nature and reduction of the displacement artifact in flow images," Magn. Reson. Med., vol. 22, no. 2, pp. 481-492, Dec. 1991.

[11] P. D. Gatehouse and D. N. Firmin, "Flow distortion and signal loss in spiral imaging," Magn. Reson. Med., vol. 41, no. 5, pp. 1023-1031, May 1999

[12] A. Sayah and A. C. Mamourian, "Flow-related artifacts in MR imaging and MR angiography of the central nervous system," Neurographics, vol. 2, no. 4, pp. 154-162, Dec. 2012.

[13] L. D. Jou, R. van Tyen, S. A. Berger, and D. Saloner, "Calculation of the magnetization distribution for fluid flow in curved vessels," Magn. Reson. Med., vol. 35, no. 4, pp. 577-584, Apr. 1996.

[14] L. D. Jou and D. Saloner, "A numerical study of magnetic resonance images of pulsatile flow in a two dimensional carotid bifurcation: A numerical study of MR images," Med. Eng. Phys., vol. 20, no. 9, pp. 643-652, Dec. 1998.

[15] P. J. Nederkoorn, Y. van der Graaf, B. C. Eikelboom, A. van der Lugt, L. W. Bartels, and W. P. Mali, "Time-of-flight MR angiography of carotid artery stenosis: Does a flow void represent severe stenosis?," Am. J. Neuroradiol., vol. 23, no. 10, pp. 1779-1784, Nov.-Dec. 2002.

[16] S. Lorthois, J. Stroud-Rossman, S. Berger, L. D. Jou, and D. Saloner, "Numerical simulation of magnetic resonance angiographies of an anatomically realistic stenotic carotid bifurcation," Ann. Biomed. Eng., vol. 33, no. 3, pp. 270-283, Jan. 2005.

[17] T. D. Nguyen, L. de Rochefort, P. Spincemaille, M. D. Cham, J. W. Weinsaft, M. R. Prince, and Y. Wang, "Effective motion-sensitizing magnetization preparation for black blood magnetic resonance imaging of the heart," J. Magn. Reson. Imag., vol. 28, no. 5, pp. 1092-1100, Nov. 2008.

[18] I. Marshall, "Computational simulations and experimental studies of 3-D phase-contrast imaging of fluid flow in carotid bifurcation geometries," J. Magn. Reson. Imag., vol. 31, no. 4, pp. 928-934, Apr. 2010.

[19] K. Perktold and M. Resch, "Numerical flow studies in human carotid artery bifurcations: Basic discussion of the geometric factor in atherogenesis," J. Biomed. Eng., vol. 12, no. 2, pp. 111-123, Mar. 1990.

[20] S. Z. Zhao, X. Y. Xu, A. D. Hughes, S. A. Thom, A. V. Stanton, B. Ari, and Q. Long, "Blood flow and vessel mechanics in a physiologically realistic model of a human carotid arterial bifurcation," J. Biomechan., vol. 33, no. 8, pp. 975-984, Aug. 2000.

[21] M. Kretowski, Y. Rolland, J. Bezy-Wendling, and J.-L. Coatrieux, "Physiologically based modeling of 3-D vascular networks and CT scan angiography," IEEE Trans. Med. Imag., vol. 22, no. 2, pp. 248-257, Feb. 2003.

[22] K. Jurczuk, M. Kretowski, and J. Bezy-Wendling, "Vascular system modeling in parallel environment-Distributed and shared memory approaches," IEEE Trans. Inf. Technol. Biomed., vol. 15, no. 4, pp. 668-672, Jul. 2011.

[23] D. Szczerba and G. Szekely, "Simulating vascular systems in arbitrary anatomies," in Lecture Notes in Computer Science. Berlin, Germany: Springer-Verlag, 2005, vol. 3750, pp. 641-648.

[24] W. Schreiner, R. Karch, M. Neumann, F. Neumann, P. Szawlowski, and S. Roedler, "Optimized arterial trees supplying hollow organs," Med. Eng. Phys., vol. 28, no. 5, pp. 416-429, Jun. 2006. 
[25] A. V. Bui, R. Manasseha, K. Liffmana, and I. D. Sutaloa, "Development of optimized vascular fractal tree models using level set distance function," Med. Eng. Phys., vol. 32, no. 7, pp. 790-794, Sep. 2010.

[26] M. H. Tawhai, A. R. Clark, and K. S. Burrowes, "Computational models of the pulmonary circulation: Insights and the move towards clinically directed studies," Pulmonary Circulation, vol. 1, no. 2, pp. 224-238, Apr.-Jun. 2011.

[27] M. Schneider, J. Reichold, B. Weber, G. Szekely, and S. Hirsch, "Tissue metabolism driven arterial tree generation," Med. Image Anal., vol. 16, no. 7, pp. 1397-1414, Oct. 2012.

[28] S. Succi, The Lattice Boltzmann Equation for Fluid Dynamics and Beyond. Oxford, U.K.: Clarendon, 2001.

[29] M. B. Reider and J. D. Sterling, "Accuracy of discrete-velocity BGK models for the simulation of the incompressible Navier-Stokes equations," Computers Fluids, vol. 24, no. 4, pp. 459-467, May 1995.

[30] M. Junk, A. Klar, and L.-S. Luo, "Asymptotic analysis of the lattice Boltzmann equation," J. Comput. Phys., vol. 210, no. 2, pp. 676-704, Dec. 2005.

[31] L. Grinberg, T. Anor, E. Cheever, J. R. Madsen, and G. E. Karniadakis, "Simulation of the human intracranial arterial tree," Phil. Trans. R. Soc. A Math. Phys. Eng. Sci., vol. 367, pp. 2371-2386, Jun. 2009.

[32] L. Grinberg, E. Cheever, T. Anor, J. R. Madsen, and G. E. Karniadakis, "Modeling blood flow circulation in intracranial arterial networks: A comparative 3-D/1-D simulation study," Ann. Biomed. Eng., vol. 39, no. 1, pp. 297-309, Jan. 2011.

[33] F. Bloch, "Nuclear induction," Phys. Rev., vol. 70, no. 7-8, pp. 460-474, Oct. 1946.

[34] J. Bittoun, J. Taquin, and M. Sauzade, "A computer algorithm for the simulation of any nuclear magnetic resonance (NMR) imaging method," Magn. Reson. Imag., vol. 2, no. 2, pp. 113-120, Jan. 1984.

[35] K. Jurczuk, M. Kretowski, P. A. Eliat, J. J. Bellanger, H. Saint-Jalmes, and J. Bezy-Wendling, "Computational modeling of MR flow imaging by the lattice Boltzmann method and Bloch equation," Magn. Reson. Imag., vol. 31, no. 7, pp. 1163-1173, Sep. 2013.

[36] M. Zamir and H. Chee, "Branching characteristics of human coronary arteries," Can. J. Physiol. Pharmacol., vol. 64, no. 6, pp. 661-668, Jun. 1986.

[37] A. Kamiya and T. Togawa, "Optimal branching structure of the vascular tree," Bull. Math. Biophys., vol. 34, no. 4, pp. 431-438, Dec. 1972.

[38] Y. C. Fung, Biomechanics: Motion, Flow, Stress and Growth.. New York: Springer, 1990

[39] D. O. Morgan, Cell Cycle: Principles of Control. Oxford, U.K.: Oxford Univ. Press, 2006.

[40] M. Kretowski, J. Bezy-Wendling, and P. Coupe, "Simulation of biphasic CT findings in hepatic cellular carcinoma by a two-level physiological model," IEEE Trans. Biomed. Eng., vol. 54, no. 3, pp. 538-542, Mar. 2007

[41] J. Bezy-Wendling and M. Kretowski, "Physiological modeling of tumor-affected renal circulation," Comput. Methods Progr. Biomed., vol. 91, no. 1, pp. 1-12, Jul. 2008.

[42] K. Jurczuk, M. Kretowski, and J. Bezy-Wendling, "Vascular network modeling-improved parallel implementation on computing cluster," in Lecture Notes in Computer Science. Berlin, U.K.: Springer-Verlag, Jul. 2010, vol. 6067, pp. 289-298.

[43] A. Grama, G. Karypis, V. Kumar, and A. Gupta, Introduction to Parallel Computing. Boston, MA: Addison-Wesley, 2003.

[44] P. Pacheco, Parallel Programming With MPI. San Francisco, CA: Morgan Kaufmann Publishers, 1997.

[45] B. Chapman, G. Jost, R. van der Pas, and D. J. Kuck, Using OpenMP. Portable Shared Memory Parallel Programming. Cambridge, MA: MIT Press, 2007.

[46] K. Jurczuk, M. Kretowski, and J. Bezy-Wendling, "Hierarchical parallel approach in vascular network modeling-Hybrid MPI+OpenMP implementation," in Lecture Notes in Computer Science. Berlin, Germany: Springer-Verlag, 2012, vol. 7203, pp. 376-385, no. 1.

[47] R. Temam, Navier-Stokes Equations: Theory and Numerical Analysis. Providence, RI: Am. Math. Soc., 2000.

[48] X. He and L.-S. Luo, "Lattice Boltzmann model for the incompressible Navier-Stokes equation,” J. Stat. Phys., vol. 88, no. 3/4, pp. 927-944, Aug. 1997.

[49] S. Chen and G. D. Doolen, "Lattice Boltzmann method for fluid flows," Annu. Rev. Fluid Mechan., vol. 30, pp. 329-364, Jan. 1998.
[50] A. M. Artoli, D. Kandhai, H. C. J. Hoefsloot, A. G. Hoekstra, and P. M. A. Sloot, "Lattice Boltzmann, a robust and accurate solver for interactive computational hemodynamics," in Lecture Notes in Computer Science. Berlin, Germany: Springer-Verlag, 2003, vol. 2657, pp. 1034-1043.

[51] X. He, G. Duckwiler, and D. J. Valentino, "Lattice Boltzmann simulation of cerebral artery hemodynamics," Comput. Fluids, vol. 38, no. 4, pp. 789-796, Apr. 2009.

[52] D. A. Wolf-Gladrow, Lattice-gas Cellular Automata and Lattice Boltzmann Models: An Introduction. Berlin, Germany: Springer-Verlang, 2000.

[53] P. L. Bhatnagar, E. P. Gross, and M. Krook, "A model for collision processes in gases. I. Small amplitude processes in charged and neutral one-component systems," Phys. Rev., vol. 94, no. 3, pp. 511-525, May 1954.

[54] R. Mei, W. Shyy, D. Yu, and L.-S. Luo, "Lattice Boltzmann method for 3-D flows with curved boundary," J. Comput. Phys., vol. 161, no. 2, pp. 680-699, Jul. 2000

[55] A. A. Mohamad, Lattice Boltzmann Method: Fundamentals and Engineering Applications with Computer Codes. New York: SpringerVerlang, 2011.

[56] Z. Guo, C. Zheng, and B. Shi, "An extrapolation method for boundary conditions in lattice Boltzmann method," Phys. Fluids, vol. 14, no. 6, pp. 2007-2010, 2002.

[57] C. Pan, J. F. Prins, and C. T. Miller, "A high-performance lattice Boltzmann implementation to model flow in porous media," Comput. Phys. Commun., vol. 158, no. 2, pp. 89-105, Apr. 2004.

[58] J. Wang, X. Zhang, A. G. Bengough, and J. W. Crawford, "Domain-decomposition method for parallel lattice Boltzmann simulation of incompressible flow in porous media," Phys. Rev. E, vol. 72, no. 1, pp. 016706-1-016706-11, Jul. 2005.

[59] H. Benoit-Cattin, G. Collewet, B. Belaroussi, H. Saint-Jalmes, and C. Odet, "The SIMRI project: A versatile and interactive MRI simulator," J. Magn. Reson., vol. 173, no. 1, pp. 97-115, Mar. 2005.

[60] T. H. Jochimsen, A. Schafer, R. Bammer, and M. E. Mosele, "Efficient simulation of magnetic resonance imaging with Bloch-Torrey equations using intra-voxel magnetization gradients," J. Magn. Reson., vol. 180, no. 1, pp. 29-38, May 2006.

[61] A. M. Aibinu, M. J. E. Salami, A. A. Shafie, and A. R. Najeeb, "MRI reconstruction using discrete Fourier transform: A tutorial," World Acad. Sci. Eng. Technol., vol. 18, pp. 179-185, 2008.

[62] R. K.-S. Kwan, A. C. Evans, and G. B. Pike, "MRI simulation-based evaluation of image processing and classification methods," IEEE Trans. Med. Imag., vol. 18, no. 11, pp. 1085-1097, Nov. 1999.

[63] J. E. Healey, P. C. Schroy, and R. Sorensen, "The intrahepatic distribution of the hepatic artery in man," J. Int. Coll. Surg., vol. 20, no. 2, pp. 133-148, Aug. 1953.

[64] C. Westbrook, C. K. Roth, and J. T. Talbot, MRI in Practice, 4th ed. Malden, MA: Wiley-Blackwell, 2011.

[65] A. J. Wheaton and M. Miyazaki, "Non-contrast enhanced MR angiography: Physical principles," J. Magn. Reson. Imag., vol. 36, no. 2, pp. 286-304, Aug. 2012.

[66] R. I. Pettigrew and W. Dannels, "Use of standard gradients with compound oblique angulation for optimal quantitative MR flow imaging in oblique vessels," Am. J. Roentgenol., vol. 148, no. 2, pp. 405-409, Feb. 1987

[67] Intel C++ Compiler 10.1 for Linux (Product In-Depth) [Online]. Available: http://software.intel.com/en-us/intel-compilers/

[68] MVAPICH: MPI over InfiniBand and iWARP [Online]. Available: mvapich. cse.ohio-state.edu

[69] C. Debbaut, D. Monbaliu, C. Casteleyn, P. Cornillie, D. Van Loo, B. Masschaele, J. Pirenne, P. Simoens, L. Van Hoorebeke, and P. Segers, "From vascular corrosion cast to electrical analog model for the study of human liver hemodynamics and perfusion," IEEE Trans. Biomed. Eng., vol. 58, no. 1, pp. 25-35, Jan. 2011.

[70] R. A. Rhoades and D. R. Bell, Medical Physiology: Principles for Clinical Medicine, 4th ed. Philadelphia, PA: Lippincott Williams Wilkin, 2012.

[71] A. C. Guyton and J. E. Hall, Textbook of Medical Physiology, 11th ed. Philadelphia, PA: Elsevier Saunders, 2005.

[72] M. A. Bernstein, K. F. King, and J. X. Zhou, Handbook of MRI Pulse Sequences. Burlington, MA: Elsevier Academic, 2004. 
[73] R. Gronas, P. G. Kalman, D. S. Kucey, and G. A. Wright, "Flow-independent angiography for peripheral vascular disease: Initial in-vivo results," J. Magn. Reson. Imag., vol. 7, no. 4, pp. 637-643, Jul. 1997.

[74] K. Krynicki, "Proton spin-lattice relaxation in pure water between $0^{\circ} \mathrm{C}$ and $100^{\circ}$ C," Physica, vol. 32, no. 1, pp. 167-178, Jan. 1966.

[75] P. Shkarin and R. G. S. Spencer, "Time domain simulation of Fourier imaging by summation of isochromats," Int. J. Imag. Syst. Technol., vol. 8, no. 5, pp. 419-426, 1997.

[76] M. Krafczyk, J. Tolke, and L.-S. Luo, "Large-eddy simulations with a multiple-relaxation-time LBE model," Int. J. Modern Phys. B, vol. 17, no. 1-2, pp. 33-39, Jan. 2003.

[77] G. M. Amdahl, "Validity of the single processor approach to achieving large scale computing capabilities," in Proc. AFIPS Conf., Atlantic City, NJ, Apr. 1967, vol. 30, pp. 483-485.

[78] S. J. Sherwin, V. Franke, J. Peiro, and K. H. Parker, "One-dimensional modelling of a vascular network in space-time variables," J. Eng. Math., vol. 47, no. 3/4, pp. 217-250, Dec. 2003.

[79] P. Reymond, F. Merenda, F. Perren, D. Rufenacht, and N. Stergiopulos, "Validation of a one-dimensional model of the systemic arterial tree," Am. J. Physiol.—Heart Circulatory Physiol., vol. 297, no. 1, pp. H208-H222, Jul. 2009.

[80] T. Anor, L. Grinberg, H. Baek, J. R. Madsen, M. V. Jayaraman, and G. E. Karniadakis, "Modeling of blood flow in arterial trees," Wiley Interdis. Rev., Syst. Biol. Med., vol. 2, no. 5, pp. 612-623, Sep. 2010.

[81] S. Varghese, S. Frankel, and P. Fischer, "Direct numerical simulation of stenotic flows. Part 2. Pulsatile flow," J. Fluid Mechan., vol. 582, pp. 281-318, Jul. 2007.
[82] S. J. Stroud, S. A. Berger, and D. Saloner, "Numerical analysis of flow through a severely stenotic carotid artery bifurcation," J. Biomechan. Eng., vol. 124, no. 1, pp. 9-20, Feb. 2002.

[83] S. J. Sherwin and G. E. Karniadakis, Spectral/Hp Element Methods for $C F D, 2$ nd ed. Oxford, U.K.: Oxford Univ. Press, 2005.

[84] L. Grinberg, V. Morozov, D. A. Fedosov, J. A. Insley, M. E. Papka, K. Kumaran, and G. E. Karniadakis, "A new computational paradigm in multiscale simulations: Application to brain blood flow," in Proc. Int. Conf. High Performance Comput., Network., Storage Anal., Seattle, WA, 2011, pp. 1-12.

[85] M. Bernaschi, M. Bisson, M. Fatica, S. Melchionna, and S. Succi, "Petaflop hydrokinetic simulations of complex flows on massive GPU clusters," Comput. Phys. Commun., vol. 184, no. 2, pp. 329-341, Feb. 2013.

[86] M. A. Castro, C. M. Putman, and J. R. Cebral, "Computational fluid dynamics modeling of intracranial aneurysms: effects of parent artery segmentation on intra-aneurysmal hemodynamics," Am. J. Neuroradiol., vol. 27, no. 8, pp. 1703-1709, Sep. 2006.

[87] S. Arcidiacono, I. V. Karlin, J. Mantzaras, and C. E. Frouzakis, "Lattice Boltzmann model for the simulation of multicomponent mixtures," Phys. Rev. E, vol. 76, no. 4, pp. 046703-1-046703-11, Oct. 2007.

[88] M. M. Dupin, I. Halliday, and C. M. Care, "A multi-component lattice Boltzmann scheme: Towards the mesoscale simulation of blood flow," Med. Eng. Phys., vol. 28, no. 1, pp. 13-18, Jan. 2006.

[89] OpenFoam [Online]. Available: http://www.openfoam.org

[90] O. Filippova and D. Hanel, "Grid refinement for lattice-BGK models," J. Computat. Phys., vol. 147, no. 1, pp. 219-228, Nov. 1998. 